\title{
Traditional Ecological Knowledge and the Cultural Significance of Plants in Hungarian Communities in Slovenia
}

Received: May 23, 2020 • Accepted: September 12, 2020

\author{
Dániel Babai - Mátyás Szépligeti - Antónia Tóth - Viktor Ulicsni \\ Institute of Ethnology, Research Centre for the Humanities, Budapest (corresponding author) - \\ Őrség National Park Directorate, Őriszentpéter - \\ Őriszentpéter - \\ Institute of Ecology and Botany, Centre for Ecological Research, Vácrátót
}

\begin{abstract}
Traditional ecological knowledge of plants is an important aspect of scholarship in relation to land use and contributes to the sustainable use and management of natural resources as well as to the monitoring of changes in the natural environment. The aim of the present paper was to examine traditional ecological knowledge in Hungarian communities in Slovenia in connection with knowledge of the plants growing in the region, their local names, and their uses. We quantified the earlier role of the utilized plant species in order to determine the former significance of certain species. We carried out structured interviews with a total of 20 individuals in three studied settlements. In the Hungarian communities in Slovenia, we uncovered knowledge of a total of 130 folk taxa. Of these, 123 taxa have local names. The majority of the folk taxa can be correlated with a single biological species. A significant proportion of the known species were utilized in some way, most of them as medicinal plants, wild edible plants, or ornamental plants. As in other farming communities, the most important species are mainly woody plants, which include the common hornbeam, the common juniper, and the silver birch. Among the herbaceous plants, utilization of the dandelion, nettle, and bulrush was significant. Members of the older generations living in the Hungarian communities in Slovenia still retain knowledge of plants that were once used on a daily basis, along with their local names and the traditional ecological knowledge connected with their earlier use.
\end{abstract}

Keywords: ethnobotany, Northeastern Slovenia, traditional ecological knowledge, use value index, salience

\section{INTRODUCTION}

Traditional ecological knowledge related to the natural flora is an important aspect of environmental knowledge in communities that directly depend on natural resources and ecosystem services. Ecosystem services necessitate a knowledge of numerous plant species. Firstly, knowledge of species is valuable from the point of view of the direct 
utilization of ecosystem services (e.g. gathering of edible or medicinal plants), while secondly, it ensures the botanical knowledge necessary for the management of natural resources (BABAI - MolNÁr 2014; GLASENAPP - THORNTON 2011). Thirdly, through the use of local, mainly species-based indicators, it contributes to the monitoring of changes in the natural environment (BERKES 2012).

In relation to folk plant knowledge, it is difficult to predict which part of the natural flora is covered by the plant knowledge of a given community. In the words of Ferenc Móra: "I believe that the Hungarians gave special names only to those plants that they made use of or suffered because of, or those that had some very striking peculiarity, while the rest were summarized under the names weeds, pests, and wild flowers" (MóRA 1960). Ethnobiologists, in line with Móra's observation, established four determining characteristics with respect to plant knowledge: 1) morphological distinctiveness (phenotypic salience); 2) striking morphological features (e.g. vividly colored flowers) (perceptual salience); 3) ecological traits (e.g. habitat indicator) (ecological salience); and 4) cultural utilization (e.g. medicinal plants) (cultural salience) (GosLer 2017; HunN 1999).

In its long history, ethnobotanical research in the Carpathian Basin has focused primarily on studies of plant species that have been utilized in some way. Research of this kind has been carried out in numerous regions of the Carpathian Basin, including Somogy (KócZián 1988, 2014); the Great Hungarian Plain (Alföld) (GrYNAEus - SzABó 1993; MolnÁR 2011); the North Hungarian Mountains (Kóczián 1984); and Southern Slovakia (Felvidék) (Kóczián - SzABó 1990); and in particular in many regions of Transylvania (review: PAPP et al. 2014a). In relation to wild edible plants, data on 236 species in the Carpathian Basin that are also biologically accurately identifiable have been published to date (review: DÉNES et al. 2012), although other types of use have also been well documented: timber use (e.g. PÉNTEK - SZABÓ 1985; TARISZnÝ́s 1978), and the utilization of wetland vegetation, such as species of bulrush (Typha spp.) (e.g. MOLNÁR 2011; SzÜCS 2003:125-126).

The principal aim of our research was to explore surviving traditional ecological knowledge - and within this, knowledge of plants - in the oral memory of Hungarianspeaking communities in Slovenia, and to examine the utilization of plants and the cultural role of environmental knowledge. We consider the documentation of environmental knowledge, as a little-researched aspect of Hungarian folk culture, to be similarly important from the point of view of the preservation of intangible cultural heritage. To the best of our knowledge, little research has been undertaken in this region to date from this particular perspective: László Kardos (1943) published ethnobotanical data in connection with folk nutrition in the Örség region, and János Bödei (1943) published data on food gathering in Göcsej, while Enikő Bazsika (2010) has published a list of folk plant names, likewise from Göcsej.

We aimed to answer the following main questions in the course of our research: 1) Which plant species growing in the wild are known and named by the Hungarianspeaking communities in the villages of Northeastern Slovenia?; 2) How are the known plant species utilized?; and 3) Which were the most important species in the region, and which species were used most diversely? 


\section{MATERIAL AND METHODOLOGY}

\section{Study area}

The studied area belongs to the Goričko region, in Northeastern Slovenia. The research was carried out in the settlements of Prosenjakovci (Pártosfalva), Motvarjevci (Szentlászló), and Središče (Szerdahely). The landscape here is typically hilly (Figure 1). The region has a temperate, continental climate, with an average annual temperature of $9.6^{\circ} \mathrm{C}$ and annual average precipitation of between 750 and $820 \mathrm{~mm}$ (KALIGARIČ et al. 2008; PAUŠ̌č et al. 2017:112). Fifty-seven percent of the territory is covered with mixed deciduous forests (sessile oak-hornbeam: Quercus petraea-Carpinetum; and beech: Fagion illyricum) or with planted pine stands, while the remainder is covered with anthropogenic hay meadows formed from cleared woodland (in dry habitats: Hypochoerido-Festucetum rupicolae, Ranunculo bulbosi-Arrhenatheretum elatioris; in mesophile habitats: AlopecuroArrhenatheretum, Anthyllido-Festucetum rubrae; and in valley bottoms: Molinion caeruleae) (15\%) and arable land (9\%) (Cousins et al. 2014:3; PAUŠIČ et al. 2017:112). This cultivated landscape is home to a rich flora, which has been protected as a nature conservation area since the foundation of the Goričko Natural Park in 2003. The number of vascular plant species discovered to date in this territory is around 1,100 .

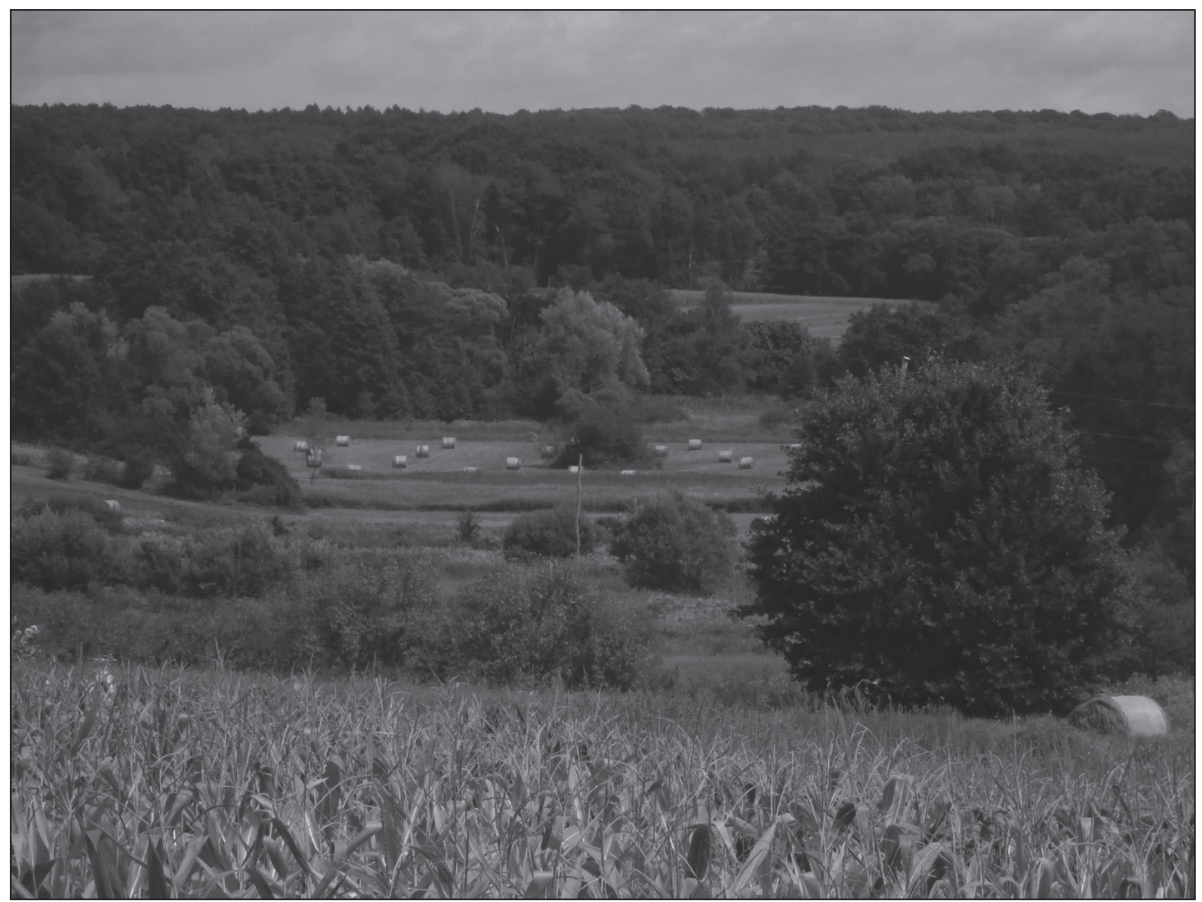

Figure 1. Characteristic landscape - a mosaic of arable land, species-rich mesophile hay meadows, and deciduous forests. Središče (Szerdahely), Slovenia, 2019. (Photo by Dániel Babai) 
A quarter of the households in the region's settlements (25.9\%) are engaged in agricultural activities exclusively on small plots (the average size of a holding is 1.59 ha, while the average number of plots is 26.7). Apart from agriculture, the majority of people are employed in the industrial and service sectors in Austria, Hungary, and other regions of Slovenia (60.7\%) (KLADNIK - REPOLUSK 1993:238). The Hungarian population is permanently decreasing in terms of both size and proportion (KLADNIK - REPOLUSK 1993:238-239).

\section{Methods}

In those communities in which extensive land use is on the decline, only a small proportion of the population are engaged in this type of agriculture, and in these communities it is primarily members of the older generations who retain memories of earlier folk plant knowledge. Based on their traditional ecological knowledge and practical experience, they know the local names of certain species, as well as the earlier ways in which they were used. For this reason, we examined the plant knowledge of the older members of the 394-strong Hungarian-speaking population of the three studied Slovenian villages mentioned above. Using the snowball sampling method, we conducted 31 semi-structured and structured interviews with a total of 20 individuals ( 7 men and 13 women). The participants had an average age of 73 (the oldest interviewee was 91 and the youngest was 54). Prior informed consent was verbally obtained from all participants, and we made audio recordings only where we had prior permission. The interviews were conducted in Hungarian.

We selected 250 species from among the approximately 1,100 vascular plant species found in the region. The selected species included both woody and herbaceous species of forest, grassland, and weed vegetation. The structured interviews were based on color photographs of the species (cf. NEwING 2010). One disadvantage of this method was that it was unsuitable for questions concerning, for example, grasses (Poaceae) and sedges (Cyperaceae). We asked about these species during the growing season, as other species characteristic of the given season. We also organized field walks with local farmers, in order to verify the photograph-based plant identifications.

In evaluating the role played by folk taxa in the life of the local community, we took into account an index based on the diversity of use, and on the frequency and exclusivity of use, drawing on the work of Turner (1988), partly adjusted to the local circumstances: the use-value index, or UVI. In relation to each individual use variable, we determined the significance of the type of use ( $\mathrm{U}$, on a five-point scale, weighted for significance of use Table 1), frequency of use (F, on a five-point scale), and the exclusivity of the species (E, on a three-point scale). The total obtained after multiplying the individual variables gives a numerical value representing the former significance of the species (the UVI):

$\mathrm{UVI}=\mathrm{U}_{1} \times \mathrm{F}_{1} \times \mathrm{E}_{1}+\mathrm{U}_{2} \times \mathrm{F}_{2} \times \mathrm{E}_{2}+\ldots+\mathrm{U}_{\mathrm{n}} \times \mathrm{F}_{\mathrm{n}} \times \mathrm{E}_{\mathrm{n}}$

In the following, we indicate the local names of the folk taxa, as well as the scientific names, in italics. With respect to the scientific names of the plants, we rely on the work of Király et al. (2009). Similarly, we indicate in italics the more important interview excerpts, which are quoted word for word. 
Table 1. Use categories and their significance (U, five-point scale, weighted for significance of use) (cf. TURNER 1988:280-281)

\begin{tabular}{|c|c|}
\hline Use categories & Use value $(\mathrm{U})$ \\
\hline Wild edible plants - e.g. roots, bulbs, tubers eaten & 4 \\
\hline Wild edible plants - e.g. stems, leaves, sprouts eaten & 4 \\
\hline Wild edible plants - e.g. flowers, fruits, seeds, nuts eaten & 4 \\
\hline Wild edible plants - e.g. children's snacks, herbs & 3 \\
\hline Wild edible plants - beverages (e.g. liquor, syrup) & 4 \\
\hline Wild edible plants - food preparation, preserving - e.g. smoking & 3 \\
\hline Medicinal plants - human - general medicine & 3 \\
\hline Medicinal plants - human - medicine for colds, coughs, etc. & 3 \\
\hline Medicinal plants - human - stomach and digestive ailments & 3 \\
\hline Medicinal plants - human - kidney and urinary ailments & 3 \\
\hline Medicinal plants - human - musculoskeletal diseases - rheumatism, muscular aches & 3 \\
\hline Medicinal plants - human - wounds, burns & 3 \\
\hline Medicinal plants - human - unspecified & 3 \\
\hline Medicinal plants - veterinary & 3 \\
\hline Primary materials - building material & 5 \\
\hline Primary materials - furniture wood & 5 \\
\hline Primary materials - firewood & 5 \\
\hline Primary materials - timber & 5 \\
\hline Farming implements - tool handles & 5 \\
\hline Farming implements - wood-splitting tools & 5 \\
\hline Farming implements - cart parts & 5 \\
\hline Farming implements - other, unspecified implements (e.g. broom, willow basket) & 4 \\
\hline Household items - kitchen tools & 2 \\
\hline Cultural significance - ritual decoration (e.g. wedding ceremonies, Christmas) & 2 \\
\hline Cultural significance - ornamental (everyday use) & 2 \\
\hline Cultural significance - children's games & 2 \\
\hline Animal fodder and bedding & 2 \\
\hline Weeds - arable weeds & 3 \\
\hline Weeds - garden weeds & 2 \\
\hline Agricultural plant protection & 3 \\
\hline Indicator species (e.g. indicators of harvesting) & 3 \\
\hline Other use types (e.g. resin, fruit tree inoculation) & 3 \\
\hline Plant species is recognised but not used specifically & 1 \\
\hline
\end{tabular}




\section{RESULTS}

\section{Characteristic features of plant knowledge}

The interviewees identified 130 plants, among which the local names of 122 folk taxa exist to this day in oral memory (Table 2). Ninety percent (110) of the named folk taxa can be identified unambiguously with a single biological species. In $10 \%$ of cases (12), the folk taxa were identified with species groups (for the most part comprising two species). The list contains a total of 17 tree species, 13 species of shrubs, 2 species of dwarf shrubs, 97 herbaceous plant species, and 1 species of liana. The known species belong to 54 families: most of them belong to the Asteraceae (15 species), Rosaceae (14 species), and Poaceae (9 species) families (Table 2).

Table 2. Folk taxa known in Hungarian communities in Slovenia, the extent to which they are known, the ways in which they are used, and their use value index (UVI). (In the case of folk taxa with several names, the names are given according to the frequency with which they were mentioned, in descending order. The number of mentions is in brackets.) Alongside the names, we have indicated the further sources of local names published in four ethnobotanical studies carried out in the region: BE: BAZsIKA (2010) (Göcsej); BJ: BöDEI (1943) (Göcsej); KL: Kardos (1943) (Örség); and TA: TótH (2009) (Örség). The utilization categories are given in the penultimate column in the table. Abbreviations: $\mathrm{MED}=$ medicinal plants, $\mathrm{EDI}=$ wild edible plants, $\mathrm{HERB}=$ aromatic herbs, $\mathrm{BEV}=$ plants used in the preparation of beverages, $\mathrm{ORN}=$ ornamental plants, FIRE=firewood, $\mathrm{CMAT}=$ construction materials, $\mathrm{TIMB}=$ sawn timber, GAME=children's games, $\mathrm{HI}=$ household implements, $\mathrm{FI}=$ farming implements, $\mathrm{HABI}=$ species that form mushroom habitats, $\mathrm{FODD}=$ fodder plants, $\mathrm{AWE}=$ arable weeds, $\mathrm{GWE}=$ garden weeds, $\mathrm{RIT}=$ ritual use, $\mathrm{PPRO}=$ plant protection, $\mathrm{DYE}=$ plants used for dyes, TRAP=species used to prepare traps. Where the number of data is fewer than 5 , we have not calculated a percentage value.

\begin{tabular}{|c|c|c|c|c|c|c|c|}
\hline Scientific name & Local name & Family & $\begin{array}{c}\text { Number } \\
\text { of data }\end{array}$ & $\begin{array}{c}\text { Extent to } \\
\text { which the } \\
\text { species } \\
\text { known\% }\end{array}$ & $\begin{array}{c}\text { Extent } \\
\text { to which } \\
\text { name is } \\
\text { known }\end{array}$ & $\begin{array}{c}\text { Type of } \\
\text { use }\end{array}$ & UVI \\
\hline $\begin{array}{c}\text { Achillea collina / } \\
\text { A. millefolium } \\
\text { egérfarkú (3), } \\
\text { cickafark (2) } \\
\text { egérfarkú } \\
\text { füjjij } / \text { kóró / } \\
\text { virág (2/1/1), } \\
\text { pulykafarok (1) }\end{array}$ & Asteraceae & 11 & 91 & 82 & $\begin{array}{c}\text { MED, } \\
\text { ORN, } \\
\text { BEV }\end{array}$ & 31 \\
\hline $\begin{array}{c}\text { Agrimonia } \\
\text { eupatoria }\end{array}$ & $\begin{array}{c}\text { bojtorján (3) } \\
\text { pulman (1), } \\
\text { cigánbua (1) }\end{array}$ & Rosaceae & 7 & 71 & 57 & MED & 18 \\
\hline $\begin{array}{c}\text { Agrostemma } \\
\text { githago }\end{array}$ & $\begin{array}{c}\text { konku (5) } \\
\text { piros konku (1), } \\
\text { konkoly (1), } \\
\text { konkó }{ }^{\mathrm{TA}}\end{array}$ & Caryophyllaceae & 8 & 88 & 75 & $\begin{array}{c}\text { AWEED, } \\
\text { ORN }\end{array}$ & 14 \\
\hline
\end{tabular}




\begin{tabular}{|c|c|c|c|c|c|c|c|}
\hline Scientific name & Local name & Family & $\begin{array}{l}\text { Number } \\
\text { of data }\end{array}$ & $\begin{array}{l}\text { Extent to } \\
\text { which the } \\
\text { species } \\
\text { known } \%\end{array}$ & $\begin{array}{l}\text { Extent } \\
\text { to which } \\
\text { name is } \\
\text { known }\end{array}$ & $\begin{array}{l}\text { Type of } \\
\text { use }\end{array}$ & UVI \\
\hline Alnus glutinosa & $\begin{array}{c}\text { egerfa }(8)^{\mathrm{BJ}, \mathrm{TA}}, \\
\text { berekfa }(2)^{\mathrm{BE}, \mathrm{TA}}, \\
\text { beregfa }^{\mathrm{BJ}}\end{array}$ & Betulaceae & 9 & 100 & 100 & $\begin{array}{l}\text { FIRE, } \\
\text { CMAT }\end{array}$ & 44 \\
\hline $\begin{array}{l}\text { Alopecurus } \\
\text { pratensis }\end{array}$ & - & Poaceae & 5 & 80 & 0 & $\begin{array}{l}\text { GAME, } \\
\text { ORN }\end{array}$ & 7 \\
\hline Amaranthus sp. & $\begin{array}{l}\text { disznóparaj (1), } \\
\text { disznóparéj (1) }\end{array}$ & Amaranthaceae & 4 & - & - & $\begin{array}{l}\text { GWE, } \\
\text { AWE }\end{array}$ & 7 \\
\hline $\begin{array}{c}\text { Ambrosia } \\
\text { artemisifolia }\end{array}$ & ambrózia (1) & Asteraceae & 2 & - & - & $\begin{array}{l}\text { GWE, } \\
\text { AWE }\end{array}$ & 5 \\
\hline $\begin{array}{l}\text { Anacamptis } \\
\text { (Orchis) morio }\end{array}$ & $\begin{array}{c}\text { kukukkvirág }(5)^{\mathrm{TA}} \text {, } \\
\text { kakukkfú (2) }\end{array}$ & Orchidaceae & 8 & 88 & 88 & ORN & 12 \\
\hline $\begin{array}{l}\text { Anagallis } \\
\text { arvensis }\end{array}$ & - $\quad\left(\right.$ esűvirág $\left.{ }^{\mathrm{TA}}\right)$ & Primulaceae & 2 & - & - & $\begin{array}{l}\text { MED, } \\
\text { GWE }\end{array}$ & 4 \\
\hline $\begin{array}{l}\text { Anemone } \\
\text { nemorosa }\end{array}$ & $\begin{array}{l}\text { csibevirág (2), } \\
\text { csillagvirág (1), } \\
\text { vadhóvirág (1) }\end{array}$ & Ranunculaceae & 10 & 100 & 40 & ORN & 4 \\
\hline $\begin{array}{c}\text { Apera spica- } \\
\text { venti }\end{array}$ & héla $(6)^{\mathrm{BE}, \mathrm{TA}}$ & Poaceae & 6 & 100 & 100 & AWE & 12 \\
\hline Arctium lappa & $\begin{array}{c}\text { lapulevél }(3)^{\mathrm{BE}, \mathrm{TA}}, \\
\text { cigántetü }(2), \\
\text { bojtorján }(1), \\
\text { bogáncs }(1), \\
\text { bojtergyán }^{\mathrm{BJ}}\end{array}$ & Asteraceae & 6 & 100 & 83 & $\begin{array}{l}\text { GAME, } \\
\text { MED }\end{array}$ & 11 \\
\hline Bellis perennis & $\begin{array}{l}\text { pipitér (5) }{ }^{\mathrm{BJ}, \mathrm{TA}}, \\
\text { Istenvirág (2), } \\
(\text { margaretta) (1) }\end{array}$ & Asteraceae & 8 & 88 & 75 & $\begin{array}{l}\text { MED, } \\
\text { EDI, } \\
\text { BEV, } \\
\text { ORN }\end{array}$ & 21 \\
\hline $\begin{array}{l}\text { Betonica } \\
\text { officinalis }\end{array}$ & - & Lamiaceae & 2 & - & - & - & 1 \\
\hline Betula pendula & nyírfa (10) & Betulaceae & 10 & 100 & 100 & $\begin{array}{l}\text { HI, FI, } \\
\text { MED, } \\
\text { ORN, } \\
\text { FIRE, } \\
\text { BEV }\end{array}$ & 76 \\
\hline $\begin{array}{c}\text { Bidens spp. } \\
\text { (perhaps Lappula } \\
\text { squarrosa) }\end{array}$ & $\begin{array}{l}\text { cigánbua (3), } \\
\text { ragadáncs }^{\mathrm{BE}}\end{array}$ & Asteraceae & 3 & - & - & $\begin{array}{l}\text { AWE, } \\
\text { GAME }\end{array}$ & 14 \\
\hline Briza media & $\begin{array}{c}\text { Mária } \\
\text { könnye }(5)^{\mathrm{BJ}}, \\
\text { máriakönny }^{\mathrm{BE}}, \\
\text { didergővirág }^{\mathrm{BJ}} \text {, } \\
\text { poloskafüjj }^{\mathrm{BJ}} \text {, } \\
\text { remegöfüjjj }^{\mathrm{BJ}} \text {, } \\
\text { remete }^{\mathrm{BJ}}, \text { tavaszi } \\
\text { zörgöfüjjj }^{\mathrm{BJ}} \text {, } \\
\text { rezgöfüjj }^{\mathrm{BE}}\end{array}$ & Poaceae & 5 & 100 & 100 & ORN & 20 \\
\hline
\end{tabular}




\begin{tabular}{|c|c|c|c|c|c|c|c|}
\hline Scientific name & Local name & Family & $\begin{array}{l}\text { Number } \\
\text { of data }\end{array}$ & $\begin{array}{l}\text { Extent to } \\
\text { which the } \\
\text { species } \\
\text { known\% }\end{array}$ & $\begin{array}{l}\text { Extent } \\
\text { to which } \\
\text { name is } \\
\text { known }\end{array}$ & $\begin{array}{l}\text { Type of } \\
\text { use }\end{array}$ & UVI \\
\hline $\begin{array}{c}\text { Bromus secalinus } \\
\text { (?) }\end{array}$ & vaduc (3) & Poaceae & 3 & - & - & $\begin{array}{l}\text { AWE, } \\
\text { EDI }\end{array}$ & 8 \\
\hline Calluna vulgaris & $\begin{array}{l}\text { vadzám (6) } \\
\text { (nyúlkóró) (1) }\end{array}$ & Ericaceae & 7 & 86 & 86 & $\begin{array}{l}\text { ORN, } \\
\text { HABI }\end{array}$ & 15 \\
\hline Caltha palustris & $\begin{array}{l}\text { gólyahír (2) }{ }^{\mathrm{TA}}, \\
\text { sárga virág (1) }\end{array}$ & Helleboraceae & 9 & 100 & 22 & ORN & 4 \\
\hline $\begin{array}{l}\text { Calystegia } \\
\text { sepium }\end{array}$ & $\begin{array}{c}\text { győjtény (6), } \\
\text { gyötin (1) } 1 \text { BE, TA } \\
\text { nagy gyöjtény (1) }\end{array}$ & Convolvulaceae & 8 & 88 & 88 & GWE & 8 \\
\hline $\begin{array}{l}\text { Campanula sp. } \\
\quad \text { (C. patula) }\end{array}$ & harangvirág $(3)^{\mathrm{TA}}$ & Campanulaceae & 6 & 67 & 50 & ORN & 1 \\
\hline $\begin{array}{l}\text { Capsella bursa- } \\
\quad \text { pastoris }\end{array}$ & $\begin{array}{l}\text { lapátlopú füjj (1), } \\
\text { vadrepce (1) }\end{array}$ & Brassicaceae & 6 & 67 & 17 & $\begin{array}{l}\text { GWE, } \\
\text { MED }\end{array}$ & 5,5 \\
\hline Carlina acaulis & - & Asteraceae & 5 & 80 & 0 & - & 2 \\
\hline $\begin{array}{l}\text { Carex spp. }(C . \\
\text { acuta, C. buekii, } \\
\text { C. elata })\end{array}$ & $\begin{array}{c}\text { sás }(6)^{\mathrm{TA}}, \text { sásfüjj } \\
(1)\end{array}$ & Cyperaceae & 7 & 100 & 100 & $\begin{array}{l}\text { FI, } \\
\text { FODD }\end{array}$ & 7,5 \\
\hline Carex brizoides & selemsás (1) & Cyperaceae & 1 & - & - & $\mathrm{HI}$ & 3 \\
\hline Carpinus betulus & $\begin{array}{l}\text { gyertyánfa }(7)^{\mathrm{TA}}, \\
\text { gyertyán }(2)^{\mathrm{TA}}\end{array}$ & Corylaceae & 9 & 100 & 100 & $\begin{array}{l}\text { FIRE, FI, } \\
\text { TIMB }\end{array}$ & 84 \\
\hline Carum carvi & $\begin{array}{c}\text { kömin }(1)^{\mathrm{BJ}, \mathrm{BE}}, \\
\text { kömény (1) }\end{array}$ & Apiaceae & 2 & - & - & HERB & 12 \\
\hline $\begin{array}{l}\text { Centaurea } \\
\text { cyanus }\end{array}$ & $\begin{array}{l}\text { búzavirág }(6)^{\mathrm{TA}}, \\
\text { kék konku }(1)^{\mathrm{BE}} \text {, } \\
\text { búzakékje (1) }\end{array}$ & Asteraceae & 7 & 100 & 100 & $\begin{array}{l}\text { AWE, } \\
\text { ORN }\end{array}$ & 8 \\
\hline $\begin{array}{l}\text { Centaurium } \\
\text { erythraea }\end{array}$ & $\begin{array}{c}\text { ezerjófüjj (1) }{ }^{\mathrm{TA}} \text {, } \\
\text { ezerjófü (6), } \\
\text { ezerfüjjj }\end{array}$ & Gentianaceae & 8 & 88 & 88 & $\begin{array}{l}\text { MED, } \\
\text { BEV }\end{array}$ & 30 \\
\hline Cerasus avium & $\begin{array}{l}\text { vadcseresnye }(6)^{\mathrm{BJ}} \\
\text { apró cseresnye (1) }\end{array}$ & Rosaceae & 6 & 100 & 100 & $\begin{array}{l}\text { EDI, } \\
\text { CMAT }\end{array}$ & 18 \\
\hline $\begin{array}{l}\text { Chelidonium } \\
\text { majus }\end{array}$ & $\begin{array}{c}\text { cinaduna }(3)^{\mathrm{B}, \mathrm{BE}}, \\
\text { tejes füjj (1) }\end{array}$ & Papaveraceae & 7 & 71 & 43 & MED & 12 \\
\hline $\begin{array}{c}\text { Chenopodium } \\
\text { album }\end{array}$ & $\begin{array}{l}\text { laboda (4), loboda } \\
\text { (2), kaszterva (1) }\end{array}$ & Chenopodiaceae & 5 & 100 & 100 & $\begin{array}{l}\text { GWE, } \\
\text { AWE }\end{array}$ & 12 \\
\hline $\begin{array}{l}\text { Cichorium } \\
\text { intybus }\end{array}$ & $\begin{array}{l}\text { hatökör-rántó füjj } \\
\text { (2), ökörfarkú } \\
\text { kóró (1), cikória } \\
\text { (1), katánkórü }\end{array}$ & Asteraceae & 6 & 100 & 67 & MED & 3 \\
\hline Cirsium arvense & $\begin{array}{l}\text { aszott (6), } \\
\text { aszottüske }^{\mathrm{BE}}\end{array}$ & Asteraceae & 6 & 100 & 100 & $\begin{array}{l}\text { AWE, } \\
\text { GWE }\end{array}$ & 14 \\
\hline $\begin{array}{l}\text { Colchicum } \\
\text { autumnale }\end{array}$ & $\begin{array}{l}\text { kikirics (2), öszi } \\
\text { kikirics (1) }\end{array}$ & Colchicaceae & 11 & 82 & 27 & - & 2 \\
\hline $\begin{array}{c}\text { Conium } \\
\text { maculatum }\end{array}$ & bürök (2) & Apiaceae & 2 & - & - & - & 4 \\
\hline
\end{tabular}




\begin{tabular}{|c|c|c|c|c|c|c|c|}
\hline Scientific name & Local name & Family & $\begin{array}{l}\text { Number } \\
\text { of data }\end{array}$ & $\begin{array}{l}\text { Extent to } \\
\text { which the } \\
\text { species } \\
\text { known } \%\end{array}$ & $\begin{array}{l}\text { Extent } \\
\text { to which } \\
\text { name is } \\
\text { known }\end{array}$ & $\begin{array}{l}\text { Type of } \\
\text { use }\end{array}$ & UVI \\
\hline $\begin{array}{l}\text { Consolida } \\
\text { regalis }\end{array}$ & szarkaláb (1) & Helleboraceae & 2 & - & - & - & 1 \\
\hline $\begin{array}{c}\text { Convallaria } \\
\text { majalis }\end{array}$ & $\begin{array}{l}\text { gyöngyvirág (7), } \\
\text { szengyörgyvirág }\end{array}$ & Convallariaceae & 7 & 100 & 100 & $\begin{array}{l}\text { ORN, } \\
\text { MED }\end{array}$ & 15 \\
\hline $\begin{array}{l}\text { Convolvulus } \\
\text { arvensis }\end{array}$ & $\begin{array}{c}\text { győjtény (6), } \\
\text { győtin }(1)^{\mathrm{BE}, \mathrm{TA}}, \\
\text { apró győjtény (1) }\end{array}$ & Convolvulaceae & 7 & 100 & 100 & GWE & 16 \\
\hline Corylus avellana & $\begin{array}{c}\text { mogyoru (3), } \\
\text { magyaru }(2)^{\mathrm{BE}, \mathrm{KL}}, \\
\text { mogyorufa (1), } \\
\text { magyarufa (1) }\end{array}$ & Corylaceae & 6 & 100 & 100 & $\begin{array}{l}\text { FI, EDI, } \\
\text { ORN, } \\
\text { GAME, } \\
\text { HI }\end{array}$ & 37 \\
\hline $\begin{array}{l}\text { Crataegus } \\
\text { monogyna }\end{array}$ & $\begin{array}{c}\text { galagonya }(4), \\
\text { sárga geregenye } \\
\text { geregöny }^{\mathrm{BE}}, \\
\text { gelegenye }^{\mathrm{KL}}\end{array}$ & Rosaceae & 9 & 78 & 44 & $\begin{array}{l}\text { MED, } \\
\text { EDI }\end{array}$ & 6 \\
\hline $\begin{array}{c}\text { Cuscuta } \\
\text { campestris }\end{array}$ & $\operatorname{kosz}(1)^{\mathrm{BE}}$ & Cuscutaceae & 5 & 40 & 20 & - & 1 \\
\hline $\begin{array}{l}\text { Dactylis } \\
\text { glomerata }\end{array}$ & - & Poaceae & 1 & - & - & FODD & 3 \\
\hline $\begin{array}{l}\text { Daphne } \\
\text { mezereum }\end{array}$ & rókaszelence (2) & Thymelaeaceae & 7 & 57 & 29 & ORN & 1 \\
\hline $\begin{array}{c}\text { Dianthus } \\
\text { carthusianorum }\end{array}$ & $\begin{array}{l}\text { vadszegfü (2), } \\
\text { vadtörökszegfü } \\
\text { (1) }\end{array}$ & Caryophyllaceae & 4 & - & - & ORN & 1 \\
\hline $\begin{array}{l}\text { Dianthus } \\
\text { superbus }\end{array}$ & vadszegfü (1) & Caryophyllaceae & 3 & - & - & ORN & 2 \\
\hline $\begin{array}{l}\text { Dryopteris spp. } \\
\text { (és Pteridium } \\
\text { aquilinum) }\end{array}$ & $\begin{array}{c}\text { pápráng (9), } \\
\text { páfrány }(2)^{\mathrm{BJ}, \mathrm{TA}}, \\
\text { paprat (1) }\end{array}$ & $\begin{array}{l}\text { Dryopteridaceae } \\
\text { s. l. (Denn } \\
\text { staedtiaceae) }\end{array}$ & 12 & 100 & 100 & $\begin{array}{l}\text { ORN, } \\
\text { FODD, } \\
\text { FI }\end{array}$ & 18 \\
\hline Elymus repens & $\begin{array}{l}\text { pörgye }(6)^{\mathrm{BE}}, \\
\text { pörje (1) }\end{array}$ & Poaceae & 6 & 100 & 100 & $\begin{array}{l}\text { GWE, } \\
\text { AWE }\end{array}$ & 18 \\
\hline $\begin{array}{l}\text { Equisetum } \\
\text { arvense }\end{array}$ & $\begin{array}{l}\text { békarokka } \\
(9)^{\mathrm{BJ}, \mathrm{BE}, \mathrm{TA}}\end{array}$ & Equisetaceae & 9 & 100 & 100 & $\begin{array}{l}\text { MED, } \\
\text { FODD }\end{array}$ & 27 \\
\hline $\begin{array}{l}\text { Eriophorum } \\
\text { latifolium, E. } \\
\text { angustifolium }\end{array}$ & $\begin{array}{l}\text { pamukfü (1), } \\
\text { pamacsfü (1), } \\
\text { sásfü (1) }\end{array}$ & Cyperaceae & 8 & 75 & 38 & ORN & 4 \\
\hline $\begin{array}{l}\text { Erythronium } \\
\text { dens-canis }\end{array}$ & $\begin{array}{c}\text { vadcikám (1), } \\
\text { vadciklámen (1) }\end{array}$ & Liliaceae & 6 & 33 & 17 & ORN & 2 \\
\hline Fagus sylvatica & bükkfa $(10)^{\mathrm{TA}}$ & Fagaceae & 10 & 100 & 100 & $\begin{array}{l}\text { FIRE, FI, } \\
\text { TIMB, } \\
\text { FODD, } \\
\text { CMAT }\end{array}$ & 41,5 \\
\hline
\end{tabular}


Dániel Babai et al.

\begin{tabular}{|c|c|c|c|c|c|c|c|}
\hline Scientific name & Local name & Family & $\begin{array}{c}\text { Number } \\
\text { of data }\end{array}$ & $\begin{array}{l}\text { Extent to } \\
\text { which the } \\
\text { species } \\
\text { known\% }\end{array}$ & $\begin{array}{l}\text { Extent } \\
\text { to which } \\
\text { name is } \\
\text { known }\end{array}$ & $\begin{array}{l}\text { Type of } \\
\text { use }\end{array}$ & UVI \\
\hline Fragaria vesca & $\begin{array}{l}\text { epörgye }(5)^{\mathrm{KL}} \text {, } \\
\text { erdei epör (1), } \\
\text { eper (2), epörke } \\
(1), \text { vadeper (1), } \\
\text { epörje }^{\mathrm{KL}}\end{array}$ & Rosaceae & 8 & 100 & 100 & EDI & 30 \\
\hline Fragaria viridis & $\begin{array}{l}\text { hársepör (1), } \\
\text { fürtös epör (1) }\end{array}$ & Rosaceae & 5 & 80 & 40 & EDI & 18 \\
\hline $\begin{array}{l}\text { Galanthus } \\
\text { nivalis }\end{array}$ & $\begin{array}{c}\text { igazi hóvirág (2), } \\
\text { rendes } \\
\text { hóvirág (2), } \\
\text { valódi hóvirág (1) }\end{array}$ & Amaryllidaceae & 5 & 100 & 100 & ORN & 1 \\
\hline $\begin{array}{c}\text { Galeobdolon } \\
\text { luteum }\end{array}$ & vadcsalán (1) & Lamiaceae & 5 & 40 & 20 & - & 1 \\
\hline $\begin{array}{l}\text { Galinsoga } \\
\text { parviflora }\end{array}$ & paprikafüjj $(1)^{\mathrm{BE}}$ & Asteraceae & 1 & - & - & GWE & 4 \\
\hline Galium aparine & - & Rubiaceae & 4 & - & - & GWE & 1 \\
\hline $\begin{array}{c}\text { Gentiana } \\
\text { pneumonanthe }\end{array}$ & encián (1) & Gentianaceae & 10 & 70 & 10 & ORN & 6 \\
\hline $\begin{array}{c}\text { Hemerocallis } \\
\text { lilio-asphodelus }\end{array}$ & $\begin{array}{l}\text { liliom (1), réti } \\
\text { liliom (1), } \\
\text { sárgaliliom }\end{array}$ & Hemerocallidaceae & 9 & 56 & 22 & ORN & 4 \\
\hline Humulus lupulus & $\begin{array}{c}\text { komló (3), } \\
\text { vadkomló (2), } \\
\text { gyöplümazzag }\end{array}$ & Cannabaceae & 5 & 80 & 80 & MED & 4 \\
\hline $\begin{array}{l}\text { Hypericum } \\
\text { perforatum }\end{array}$ & $\begin{array}{l}\text { csillagvirág (3), } \\
\text { vasvirág (1) }\end{array}$ & Hypericaceae & 6 & 100 & 67 & MED & 9 \\
\hline Iris pseudacorus & $\begin{array}{l}\text { sárga liliom (6), } \\
\text { liliom (1), } \\
\text { vízililijom }^{\mathrm{BE}}\end{array}$ & Iridaceae & 10 & 90 & 70 & ORN & 4 \\
\hline Iris sibirica & $\begin{array}{c}\text { írisz (2), kékliliom } \\
\text { (2), vadírisz (1) }\end{array}$ & Iridaceae & 8 & 63 & 63 & ORN & 2 \\
\hline Juncus effusus & $\begin{array}{l}\text { zsombék (4), } \\
\text { zsombik (2) }\end{array}$ & Juncaceae & 6 & 100 & 100 & $\begin{array}{l}\text { GAME, } \\
\text { ORN, HI }\end{array}$ & 18 \\
\hline $\begin{array}{l}\text { Juniperus } \\
\text { communis }\end{array}$ & $\begin{array}{c}\text { pattagu (9), } \\
\text { boróka (3), } \\
\text { pattogó } \\
\text { borosin }{ }^{\mathrm{BJ}, \mathrm{BE}}, \\
\text { pattogu borosán } \\
\text { borosánfenyü }{ }^{\mathrm{KL}} \\
\end{array}$ & Cupressaceae & 9 & 100 & 100 & $\begin{array}{l}\text { HERB, } \\
\text { FI, MED, } \\
\text { BEV }\end{array}$ & 89 \\
\hline $\begin{array}{l}\text { Lamium } \\
\text { purpureum }\end{array}$ & $\begin{array}{c}\text { vadcsalán (6), } \\
\text { árvacsalán (2), } \\
\text { vadcsalánt }{ }^{\mathrm{TA}}\end{array}$ & Lamiaceae & 8 & 100 & 75 & $\begin{array}{l}\text { EDI, } \\
\text { GWE, } \\
\text { MED }\end{array}$ & 12,5 \\
\hline $\begin{array}{c}\text { Leucanthemum } \\
\text { vulgare }\end{array}$ & $\begin{array}{l}\text { margaréta (4), } \\
\text { vadpipitér (1) }\end{array}$ & Asteraceae & 4 & - & - & MED & 1,5 \\
\hline
\end{tabular}


Traditional Ecological Knowledge and the Cultural Significance of Plants... 491

\begin{tabular}{|c|c|c|c|c|c|c|c|}
\hline Scientific name & Local name & Family & $\begin{array}{l}\text { Number } \\
\text { of data }\end{array}$ & $\begin{array}{l}\text { Extent to } \\
\text { which the } \\
\text { species } \\
\text { known\% }\end{array}$ & $\begin{array}{l}\text { Extent } \\
\text { to which } \\
\text { name is } \\
\text { known }\end{array}$ & $\begin{array}{l}\text { Type of } \\
\text { use }\end{array}$ & UVI \\
\hline Leucojum verum & $\begin{array}{c}\text { hóvirág (10), } \\
\text { tőzike (4), } \\
\text { gyöngyvirág (1) }\end{array}$ & Amaryllidaceae & 11 & 100 & 100 & $\begin{array}{l}\text { ORN, } \\
\text { IND }\end{array}$ & 22 \\
\hline $\begin{array}{l}\text { Ligustrum } \\
\text { vulgare }\end{array}$ & vadorgona (1) & Olaeaceae & 4 & - & - & ORN & 1 \\
\hline Linaria vulgaris & $\begin{array}{c}\text { vizestetű }(1), \\
\text { vadtátika }(1)^{\mathrm{BE}}, \\
\text { vizestetüfüjjj }^{\mathrm{BJ}}\end{array}$ & Scrophulariaceae & 5 & 100 & 20 & MED & 1,5 \\
\hline $\begin{array}{c}\text { Lotus } \\
\text { corniculatus }\end{array}$ & $\begin{array}{c}\text { szarvaskeret (3), } \\
\text { vadszarvas (1), } \\
\text { szarvaskerep- } \\
\text { lucerna (1) }\end{array}$ & Fabaceae & 5 & 100 & 100 & FODD & 24 \\
\hline $\begin{array}{l}\text { Lychnis flos- } \\
\quad \text { cuculi }\end{array}$ & $\begin{array}{l}\text { fecskevirág } \\
(6)^{\mathrm{BE}, \mathrm{TA}}, \\
\text { fecskefarkú (2), } \\
\text { fecskefü (1) }\end{array}$ & Caryophyllaceae & 11 & 100 & 82 & ORN & 6 \\
\hline $\begin{array}{l}\text { Lycopodium } \\
\text { clavatum }\end{array}$ & $\begin{array}{c}\text { judapor (8), } \\
\text { variláb (1), } \\
\text { nyúlmadzag (1) }\end{array}$ & Lycopodiaceae & 11 & 100 & 82 & $\begin{array}{l}\text { MED, } \\
\text { GAME }\end{array}$ & 32 \\
\hline $\begin{array}{l}\text { Lythrum } \\
\text { salicaria }\end{array}$ & $\begin{array}{c}\text { kígyópásztorfüjj } \\
\text { (1) }\end{array}$ & Lythraceae & 9 & 78 & 11 & ORN & 1 \\
\hline Malus sylvestris & vadalma $(4)^{\mathrm{BJ}}$ & Rosaceae & 5 & 80 & 80 & $\begin{array}{l}\text { EDI, } \\
\text { HERB }\end{array}$ & 22 \\
\hline $\begin{array}{c}\text { Malva neglecta, } \\
\text { M. sylvestris }\end{array}$ & $\begin{array}{c}\text { papsajtú füjj (1), } \\
\text { papsajtfüjjKL }\end{array}$ & Malvaceae & 5 & 60 & 20 & EDI & 3 \\
\hline $\begin{array}{l}\text { Matricaria } \\
\text { recutita (ritkán } \\
\text { M. discoidea) }\end{array}$ & $\begin{array}{l}\text { pipitér (2), } \\
\text { kamilla }^{\mathrm{TA}}\end{array}$ & Asteraceae & 2 & - & - & MED & 6 \\
\hline $\begin{array}{c}\text { Myosotis } \\
\text { nemorosa, } M . \\
\text { palustris }\end{array}$ & $\begin{array}{c}\text { nefelejcs (6), } \\
\text { kéknefelejcs (3) }\end{array}$ & Boraginaceae & 6 & 100 & 100 & ORN & 16 \\
\hline Nardus stricta & $\begin{array}{l}\text { sörtél (1), } \\
\text { drótfü (1) }\end{array}$ & Poaceae & 4 & 50 & 50 & - & 6 \\
\hline Padus avium & $\begin{array}{l}\text { szelence }(4)^{\mathrm{KL}}, \\
\text { szelencefa }(2), \\
\text { vadszelence }(1)^{\mathrm{KL}}\end{array}$ & Rosaceae & 7 & 100 & 100 & $\begin{array}{l}\text { MED, } \\
\text { ORN, } \\
\text { EDI }\end{array}$ & 10 \\
\hline Papaver rhoeas & pipacs $(6)^{\mathrm{B}, \mathrm{TA}}$ & Papaveraceae & 6 & 100 & 100 & $\begin{array}{l}\text { AWE, } \\
\text { ORN }\end{array}$ & 13 \\
\hline $\begin{array}{c}\text { Persicaria } \\
\text { maculosa }\end{array}$ & $\begin{array}{l}\text { keserüfüjj (2), } \\
\text { köserüfüjj (1) }\end{array}$ & Polygonaceae & 3 & - & - & $\begin{array}{l}\text { AWE, } \\
\text { GWE }\end{array}$ & 8 \\
\hline $\begin{array}{l}\text { Petasites } \\
\text { hybridus }\end{array}$ & - & Asteraceae & 3 & - & - & GAME & 1 \\
\hline $\begin{array}{l}\text { Phragmites } \\
\text { australis }\end{array}$ & nád $(1)^{\mathrm{BJ}, \mathrm{TA}}$ & Poaceae & 1 & - & - & - & 1 \\
\hline
\end{tabular}




\begin{tabular}{|c|c|c|c|c|c|c|c|}
\hline Scientific name & Local name & Family & $\begin{array}{l}\text { Number } \\
\text { of data }\end{array}$ & $\begin{array}{l}\text { Extent to } \\
\text { which the } \\
\text { species } \\
\text { known\% }\end{array}$ & $\begin{array}{l}\text { Extent } \\
\text { to which } \\
\text { name is } \\
\text { known }\end{array}$ & $\begin{array}{l}\text { Type of } \\
\text { use }\end{array}$ & UVI \\
\hline Picea abies & $\begin{array}{c}\text { bürkösfenyő (4), } \\
\text { bürkösfa (4), } \\
\text { fürtös fenyő (2), } \\
{\text { karácsonyfa }(1)^{\mathrm{TA}}}^{\text {lucfenyö }}{ }^{\mathrm{TA}} \\
\end{array}$ & Pinaceae & 9 & 100 & 100 & $\begin{array}{l}\text { RIT, } \\
\text { FODD, } \\
\text { ORN, } \\
\text { MED }\end{array}$ & 35 \\
\hline Pinus sylvestris & $\begin{array}{c}\text { fenyőfa (7), } \\
\text { fenyő }(1)^{\mathrm{TA}}, \\
\text { erdei fenyö }(1)^{\mathrm{TA}}\end{array}$ & Pinaceae & 9 & 100 & 100 & $\begin{array}{l}\text { CMAT, } \\
\text { MED, } \\
\text { ORN, } \\
\text { TIMB }\end{array}$ & 55 \\
\hline $\begin{array}{l}\text { Plantago } \\
\text { lanceolata }\end{array}$ & $\begin{array}{l}\text { kígyónyelvű } \\
\text { füjjj (4) }{ }^{\mathrm{BJ}, \mathrm{BE}}, \\
\text { szúklevelü } \\
\text { útifüjj (1) }\end{array}$ & Plantaginaceae & 8 & 100 & 63 & MED & 6 \\
\hline Plantago major & útifüjj $(5)^{\mathrm{TA}}$ & Plantaginaceae & 5 & 100 & 100 & $\begin{array}{l}\text { MED, } \\
\text { GAME }\end{array}$ & 20 \\
\hline $\begin{array}{l}\text { Polygonatum } \\
\text { latifolium }\end{array}$ & $\begin{array}{c}\text { sarkantyúfü (1), } \\
\text { sarkantyúvirág (1) }\end{array}$ & Convallariaceae & 6 & 67 & 17 & - & 2 \\
\hline $\begin{array}{l}\text { Polygonum } \\
\text { aviculare }\end{array}$ & $\begin{array}{l}\text { porcogós fü (1), } \\
\text { kövecsfüjj (1) }\end{array}$ & Polygonaceae & 4 & - & - & $\begin{array}{l}\text { GWE, } \\
\text { FODD }\end{array}$ & 8 \\
\hline Populus tremula & $\begin{array}{c}\text { nyárfa (3), } \\
\text { vadnyárfa (2), } \\
\text { fekete nyár (1) }\end{array}$ & Salicaceae & 6 & 100 & 83 & FIRE, HI & 16 \\
\hline Primula vulgaris & $\begin{array}{l}\text { sipulóvirág } \\
(10)^{\mathrm{BE}, \mathrm{TA}}, \\
\text { zsibavirág }^{\mathrm{BE}}\end{array}$ & Primulaceae & 10 & 100 & 100 & $\begin{array}{l}\text { MED, } \\
\text { GAME, } \\
\text { BEV }\end{array}$ & 27 \\
\hline Prunus spinosa & $\begin{array}{l}\text { gereginye- } \\
\text { tüske }(8), \\
\text { kökény }(5)^{\mathrm{BJ}} \text {, } \\
\text { kökin }^{\mathrm{KL}}\end{array}$ & Rosaceae & 8 & 100 & 100 & $\begin{array}{c}\text { EDI, } \\
\text { MED, FI }\end{array}$ & 21 \\
\hline $\begin{array}{c}\text { Pteridium } \\
\text { aquilinum és } \\
\text { Dryopteris spp. }\end{array}$ & $\begin{array}{c}\text { pápráng }(9), \\
\text { páfrány }(2)^{\mathrm{BJ}, \mathrm{TA}}, \\
\text { paprat (1) }\end{array}$ & $\begin{array}{c}\text { Denn staedtiaceae } \\
\text { (Dryopteridaceae } \\
\text { s.l.) }\end{array}$ & 12 & 100 & 100 & $\begin{array}{l}\text { ORN, } \\
\text { FODD, } \\
\text { FI }\end{array}$ & 18 \\
\hline $\begin{array}{l}\text { Pulmonaria } \\
\text { officinalis }\end{array}$ & tüdővirág (1) & Boraginaceae & 2 & - & - & ORN & 2 \\
\hline Pyrus pyraster & vadkörte $(5)^{\mathrm{BJ}}$ & Rosaceae & 5 & 100 & 100 & $\begin{array}{l}\text { EDI, } \\
\text { BEV }\end{array}$ & 12 \\
\hline $\begin{array}{l}\text { Quercus petraea } \\
\text { agg., Q. robur }\end{array}$ & $\begin{array}{l}\text { tölgyfa }(8)^{\mathrm{TA}}, \\
\text { tölfa }(3)^{\mathrm{BE}}, \\
\text { csepefa }^{\mathrm{BJ}, \mathrm{BE}}\end{array}$ & Fagaceae & 10 & 100 & 100 & $\begin{array}{l}\text { FODD, } \\
\text { EDI }\end{array}$ & 64 \\
\hline $\begin{array}{l}\text { Ranunculus } \\
\text { acris, R. repens }\end{array}$ & sárga virág (3) & Ranunculaceae & 8 & 100 & 38 & $\begin{array}{l}\text { IND, } \\
\text { FODD }\end{array}$ & 16 \\
\hline $\begin{array}{c}\text { Robinia } \\
\text { pseudoacacia }\end{array}$ & $\begin{array}{l}\text { agácfa }(4)^{\mathrm{BJ}, \mathrm{BE}}, \\
\text { agác }(2), \\
\text { akácfa }(2)\end{array}$ & Fabaceae & 7 & 100 & 100 & $\begin{array}{l}\text { FIRE, FI, } \\
\text { MED, } \\
\text { EDI, } \\
\text { BEV, } \\
\text { FODD }\end{array}$ & 60 \\
\hline
\end{tabular}




\begin{tabular}{|c|c|c|c|c|c|c|c|}
\hline Scientific name & Local name & Family & $\begin{array}{l}\text { Number } \\
\text { of data }\end{array}$ & $\begin{array}{l}\text { Extent to } \\
\text { which the } \\
\text { species } \\
\text { known \% }\end{array}$ & $\begin{array}{l}\text { Extent } \\
\text { to which } \\
\text { name is } \\
\text { known }\end{array}$ & $\begin{array}{l}\text { Type of } \\
\text { use }\end{array}$ & UVI \\
\hline Rosa canina agg. & $\begin{array}{c}\text { csipkebogyó (6), } \\
\text { tüskebogyó (3), } \\
\text { vadtüskerózsa (2), } \\
\text { vadrózsa (2), } \\
\text { seggibugya (2) } \\
\text { csipkebogyu (2), }^{\mathrm{BE}} \text {, } \\
\text { csicskenye }^{\mathrm{BE}}\end{array}$ & Rosaceae & 9 & 100 & 89 & $\begin{array}{l}\text { MED, } \\
\text { EDI, } \\
\text { GAME }\end{array}$ & 32 \\
\hline Rosa gallica & $\begin{array}{c}\text { törpe } \\
\text { vadtüskerózsa (1), } \\
\text { vadtüskerózsa (1), } \\
\text { csicskënye }^{\mathrm{BJ}, \mathrm{KL}} \\
\text { csipkënye }^{\mathrm{KL}}\end{array}$ & Rosaceae & 3 & - & - & ORN & 2 \\
\hline $\begin{array}{l}\text { Rubus fruticosus } \\
\text { agg. }\end{array}$ & $\begin{array}{l}\text { tüskeszeder }(7)^{\mathrm{KL}} \text {, } \\
\text { vadszeder }(1) \text {, } \\
\text { tüskisszeder }{ }^{\mathrm{BE}} \text {, } \\
\text { szedernye }^{\mathrm{KL}}\end{array}$ & Rosaceae & 8 & 100 & 100 & $\begin{array}{l}\text { EDI, } \\
\text { BEV }\end{array}$ & 15 \\
\hline Rubus idaeus & $\begin{array}{l}\text { vadmálna (1), } \\
\text { málna }{ }^{\mathrm{KL}}, \\
\text { himpiér }^{\mathrm{KL}}\end{array}$ & Rosaceae & 3 & - & - & EDI & 3 \\
\hline $\begin{array}{c}\text { Rumex } \\
\text { obtusifolius }\end{array}$ & $\begin{array}{l}\text { kásás füjj (4), } \\
\text { lósóska (4) }{ }^{\mathrm{BE}}, \\
\text { nyúlsóska (2), } \\
\text { köserü füjj (1) }\end{array}$ & Polygonaceae & 8 & 100 & 88 & $\begin{array}{l}\text { MED, } \\
\text { FODD }\end{array}$ & 15 \\
\hline Salix alba & $\begin{array}{c}\text { sípfa (2), } \\
\text { sípfabokor (1), } \\
\text { cicamaca (1), } \\
\text { füzfa (1) }\end{array}$ & Salicaceae & 4 & - & - & - & 2 \\
\hline Salix caprea & $\begin{array}{c}\text { rakodla (2), } \\
\text { lakotla (1), } \\
\text { lakotla-bokor (1), } \\
\text { cicamacuka (1), } \\
\text { cicamaca }^{\mathrm{BE}}, \\
\text { zsibásmacuka }^{\mathrm{BE}} \\
\text { (a barka), } \\
\text { rakottlafa }^{\mathrm{BJ}}, \\
\text { rakotla }^{\mathrm{BE}}\end{array}$ & Salicaceae & 6 & 67 & 67 & HI & 6 \\
\hline Salix fragilis & füzfa (4), sípfa (1) & Salicaceae & 4 & - & - & $\begin{array}{l}\text { ORN, } \\
\text { GAME }\end{array}$ & 4 \\
\hline Salix viminalis & $\begin{array}{c}\text { szíjács (3), } \\
\text { vidra (1), } \\
\text { vidrabokor (1) }\end{array}$ & Salicaceae & 5 & 100 & 100 & HI & 32 \\
\hline Sambucus ebulus & földi bodza ${ }^{\mathrm{BJ}}$ & Caprifoliaceae & 1 & - & - & - & 2 \\
\hline Sambucus nigra & $\begin{array}{l}\text { bodza }(3)^{\mathrm{BJ}, \mathrm{BE}, \mathrm{TA}} \\
\quad \text { bozda }(1)\end{array}$ & Caprifoliaceae & 4 & - & - & $\begin{array}{l}\text { MED, } \\
\text { EDI, } \\
\text { BEV }\end{array}$ & 12 \\
\hline $\begin{array}{l}\text { Sanguisorba } \\
\text { officinalis }\end{array}$ & $\begin{array}{c}\text { birkabogározó } \\
\text { fü (1), } \\
\text { (pénzesfü) (1) }\end{array}$ & Rosaceae & 12 & 75 & 8 & GAME & 4 \\
\hline
\end{tabular}


Dániel Babai et al.

\begin{tabular}{|c|c|c|c|c|c|c|c|}
\hline Scientific name & Local name & Family & $\begin{array}{c}\text { Number } \\
\text { of data }\end{array}$ & $\begin{array}{l}\text { Extent to } \\
\text { which the } \\
\text { species } \\
\text { known } \%\end{array}$ & $\begin{array}{l}\text { Extent } \\
\text { to which } \\
\text { name is } \\
\text { known }\end{array}$ & $\begin{array}{l}\text { Type of } \\
\text { use }\end{array}$ & UVI \\
\hline Setaria viridis & $\begin{array}{l}\text { mohár (1), } \\
\text { kásás füjj (1) }\end{array}$ & Poaceae & 2 & - & - & - & 1 \\
\hline Stellaria media & tikhúr (5) & Caryophyllaceae & 5 & 100 & 100 & $\begin{array}{l}\text { GWE, } \\
\text { EDI, } \\
\text { FODD }\end{array}$ & 12 \\
\hline $\begin{array}{l}\text { Symphytum } \\
\text { officinalis }\end{array}$ & nadálytő (1) & Boraginaceae & 5 & 80 & 40 & $\begin{array}{l}\text { EDI, } \\
\text { PPRO }\end{array}$ & 3 \\
\hline $\begin{array}{l}\text { Tanacetum } \\
\text { vulgare }\end{array}$ & $\begin{array}{c}\text { feketekoró }(2)^{\mathrm{BJ}} \text {, } \\
\text { kóró }(1), \\
\text { grádicskóró } \\
\text { mögyekóru }^{\mathrm{BE}}\end{array}$ & Asteraceae & 4 & - & - & ORN & 2 \\
\hline $\begin{array}{l}\text { Taraxacum } \\
\text { officinale agg. }\end{array}$ & $\begin{array}{c}\text { kákics }(6)^{\mathrm{BJ}, \mathrm{KL}, \mathrm{TA}}, \\
\text { gyermekláncfü } \\
(4)^{\mathrm{KL}}, \text { láncfü }(2)\end{array}$ & Asteraceae & 7 & 100 & 86 & $\begin{array}{l}\text { EDI, } \\
\text { MED, } \\
\text { FODD, } \\
\text { GAME }\end{array}$ & 49 \\
\hline Tilia cordata & hársfa $(2)^{\mathrm{B}, \mathrm{TA}}$ & Tiliaceae & 2 & - & - & MED & 3 \\
\hline $\begin{array}{l}\text { Trifolium } \\
\text { pratense }\end{array}$ & $\begin{array}{c}\text { piros lóher (3), } \\
\text { fekete lóher (1), } \\
\text { lúher (1), } \\
\text { vadlóher }^{\mathrm{TA}}\end{array}$ & Fabaceae & 4 & - & - & $\begin{array}{l}\text { FODD, } \\
\text { MED }\end{array}$ & 21 \\
\hline Trifolium repens & $\begin{array}{l}\text { fehér lóher (7), } \\
\text { vadlóher (1) } \\
\text { lóher (1) }\end{array}$ & Fabaceae & 8 & 100 & 100 & $\begin{array}{l}\text { FODD, } \\
\text { PPRO }\end{array}$ & 18 \\
\hline Typha latifolia & $\begin{array}{l}\text { botika }(4)^{\mathrm{BE}}, \\
\text { gyékény }(3)^{\mathrm{TA}}, \\
\text { gyékén }(3), \\
\text { sás }(3), \text { nád }(1)\end{array}$ & Typhaceae & 7 & 100 & 100 & ORN, FI & 36 \\
\hline Urtica dioica & $\begin{array}{l}\text { csalán (6), } \\
\text { csollán }{ }^{\mathrm{BJ}}\end{array}$ & Urticaceae & 6 & 100 & 100 & $\begin{array}{l}\text { MED, } \\
\text { EDI, } \\
\text { PPRO }\end{array}$ & 39 \\
\hline $\begin{array}{l}\text { Vaccinium } \\
\text { myrtillus }\end{array}$ & farkasszőlő (1) & Ericaceae & 2 & - & - & $\begin{array}{l}\text { MED, } \\
\text { BEV }\end{array}$ & 6 \\
\hline Viburnum opulus & $\begin{array}{c}\text { farkascseresnye } \\
\text { (1) }\end{array}$ & Caprifoliaceae & 2 & - & - & ORN & 2 \\
\hline Vicia cracca & $\begin{array}{c}\text { vadlencse (4), } \\
\text { lucerna (1), } \\
\text { bükkönyvirág (1) }\end{array}$ & Fabaceae & 6 & 83 & 67 & AWE & 3 \\
\hline Vinca minor & $\begin{array}{c}\text { metring }(6)^{\mathrm{TA}}, \\
\text { zöld metring }(1), \\
\text { téliződ }^{\mathrm{BE}}\end{array}$ & Apocynaceae & 7 & 100 & 100 & $\begin{array}{l}\text { ORN, } \\
\text { MED }\end{array}$ & 15 \\
\hline Viola arvensis & vadárvácska (6) & Violaceae & 6 & 100 & 100 & - & 2 \\
\hline Viola odorata & $\begin{array}{l}\text { szagos ibolya (6), } \\
\text { ibolya }(1)^{\mathrm{BE}}, \\
\text { rendes ibolya }(1)\end{array}$ & Violaceae & 8 & 100 & 100 & $\begin{array}{l}\text { ORN, } \\
\text { BEV, } \\
\text { DYE }\end{array}$ & 16 \\
\hline
\end{tabular}




\begin{tabular}{|c|c|c|c|c|c|c|c|}
\hline Scientific name & Local name & Family & $\begin{array}{c}\text { Number } \\
\text { of data }\end{array}$ & $\begin{array}{c}\text { Extent to } \\
\text { which the } \\
\text { species } \\
\text { known\% }\end{array}$ & $\begin{array}{c}\text { Extent } \\
\text { to which } \\
\text { name is } \\
\text { known }\end{array}$ & $\begin{array}{c}\text { Type of } \\
\text { use }\end{array}$ & UVI \\
\hline $\begin{array}{c}\text { Viola } \\
\text { reichenbachiana, } \\
\text { Viola spp. }\end{array}$ & $\begin{array}{c}\text { vadibolya (5), } \\
\text { ibolya (5) } \\
\text { kékibolya (2) }\end{array}$ & Violaceae & 10 & 100 & 100 & $\begin{array}{c}\text { MED, } \\
\text { ORN, } \\
\text { BEV }\end{array}$ & 15 \\
\hline $\begin{array}{c}\text { Viscum album } \\
\text { /Loranthus } \\
\text { europaeus }\end{array}$ & fagyöngy (6) & $\begin{array}{c}\text { Loranthaceae (incl. } \\
\text { Viscaceae) }\end{array}$ & 6 & 100 & 100 & $\begin{array}{c}\text { MED, } \\
\text { ORN, } \\
\text { TRAP }\end{array}$ & 19 \\
\hline
\end{tabular}

\section{The most important uses of the known species}

In terms of knowledge of folk taxa and biological species, there is a significant emphasis on utilization. With respect to the 130 known folk taxa, a total of 218 data items referring to utilization were mentioned during the interviews. In relation to most species, utilization as ornamental plants (in vases or bouquets) (46 species), use of medicinal plants for human and animal health (39 species), and the consumption of wild edible plants (39 species) were mentioned. In the case of 11 of the mentioned folk taxa, based on the currently available data there was no information regarding utilization (e.g. Galeobdolon luteum, Phragmites australis, Viola arvensis) (Table 3).

Table 3. Folk taxa used in Hungarian communities in Slovenia. The number of times they were mentioned is given in brackets

\begin{tabular}{|c|c|c|}
\hline Type of use & $\begin{array}{l}\text { Number of } \\
\text { species }\end{array}$ & Most important species \\
\hline Medicinal plants & 39 & $\begin{array}{l}\text { e.g. Lycopodium clavatum (11), Achillea spp., Rosa canina agg. (7- } \\
\text { 7), Centaurium erythreae, Equisetum arvense, Viscum album (5-5), } \\
\text { Agrimonia eupatoria, Chelidonium majus, Hypericum perforatum, } \\
\text { Plantago major, Urtica dioica (4-4). }\end{array}$ \\
\hline Wild edible plants & 39 & $\begin{array}{l}\text { e.g. Fragaria vesca (8), Rosa canina (5), Rubus caesius (6), Taraxacum } \\
\text { officinale agg. (5), Fragaria viridis, Prunus spinosa (4-4). }\end{array}$ \\
\hline Timber products & 3 & Carpinus betulus, Fagus sylvatica, Pinus sylvestris \\
\hline Firewood & 6 & $\begin{array}{l}\text { Alnus glutinosa, Betula pendula, Carpinus betulus, Fagus sylvatica, } \\
\text { Populus tremula, Robinia pseudo-acacia. }\end{array}$ \\
\hline Fodder plants & 15 & $\begin{array}{l}\text { e.g. good for fodder: Quercus spp. (6), Lotus corniculatus (5), } \\
\text { Trifolium repens és T. pratense (3-3), Robinia pseudoacacia, Picea } \\
\text { abies, Taraxacum officinale, Stellaria media (1-1); bad for fodder: } \\
\text { Equisetum arvense, Carex spp., Ranunculus spp. (1-1). }\end{array}$ \\
\hline Farming implements & 10 & $\begin{array}{l}\text { Carpinus betulus (5), Betula pendula (4), Corylus avellana (3), } \\
\text { Dryopteris spp., Fagus sylvatica, Robinia pseudoacacia (1-1) }\end{array}$ \\
\hline Household implements & 10 & $\begin{array}{l}\text { Betula pendula (7), Juniperus communis (5), Salix viminalis (5), Salix } \\
\text { caprea, Typha angustifolia (3) Corylus avellana, Populus tremula, } \\
\text { Carex spp. Juncus effusus, Prunus spinosa (1-1). }\end{array}$ \\
\hline Ornamental plants & 46 & $\begin{array}{l}\text { e.g. Briza media (5), Myosotis sp. (5), Leucojum vernalis, Typha } \\
\text { angustifolia, Vinca minor, Viola odorata (4-4). }\end{array}$ \\
\hline Ritual use & 1 & Picea abies (4), Betula pendula (1) \\
\hline
\end{tabular}




\section{The most important and most diversely utilized folk taxa}

Based on the use-value index (UVI) of the 130 folk taxa, and taking into account 1) the diversity; and 2) frequency of their utilization; and 3) the exclusivity of the species' utilization, the most important species (key cultural species) for the Hungarian communities in Slovenia were woody plants (the first six of the species with the highest UVI were woody species). The most important of the woody key species in the region were: the common hornbeam (Carpinus betulus [UVI: 84]); the common juniper (Juniperus communis [79]), and the silver birch (Betula pendula [74.5]). Among the herbaceous plants, the dandelion (Taraxacum officinale agg. [UVI: 49]), the common nettle (Urtica dioica [39]), and the broadleaf cattail (bulrush) (Typha latifolia [36]) were the most important (Table 4).

\section{Other important and valuable uses}

Information connected to therapeutic uses in humans and in veterinary health was mentioned in relation to 39 species. Some species were collected in large quantities for selling in bulk, and this was typically a source of income for children. Occasionally, species that were not even used locally were collected, such as lilies of the valley (Convallaria majalis), for example: "when I was a little girl, we used to collect the leaves, after it had flowered, and take them home in a wheelbarrow. They could be sold. / BD: Did you use them at home as well? / Well, maybe in a vase at home, or at the cemetery, otherwise no."

Children would eat the nectar from the flowers of the white deadnettle (Lamium album) as a treat: "when we were little, we used to pick the flowers and suck them, they were so sweet.".

Decorative uses (bouquets, dried flowers) were mentioned in connection with 46 species. Among the grass species, mention was made of the use of quaking grass (Briza media) as a dried flower, while in the course of our fieldwork we saw purple moor-grass (Molinia caerulea) in a vase (no local name) (Figure 2c).

Numerous species of colorful meadow flowers were picked to put in vases. Among them, the use of various species of Myosotis can be highlighted: these were not only picked for vases, but were used to create specific arrangements: "you put some cold water in a plate, then you arrange them, one by one, round the edge. Then they stand up by themselves. You can put a smaller vase of forget-me-nots in the middle... It's really pretty." ${ }^{3}$

1 Interviewee G (woman, 1941, Prosenjakovci [Pártosfalva]). Interview was conducted by Daniel Babai (13 08 2019).

2 Interviewee A (woman, 1943, Motvarjevci [Szentlászló]). Interview was conducted by Daniel Babai (1308 2019).

3 Interviewee L (woman, 1933, Motvarjevci [Szentlászló]). Interview was conducted by Daniel Babai (1308 2019). 


\begin{tabular}{|c|c|c|c|c|c|c|}
\hline 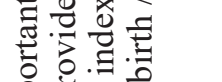 & 5 & $\approx$ & m & $\simeq$ & $\infty$ & $\varpi$ \\
\hline & س & $N$ & $N$ & - & - & \\
\hline & 工 & + & + & $m$ & N & \\
\hline & 3 & $\nabla$ & $\nabla$ & 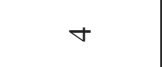 & $\nabla$ & \\
\hline 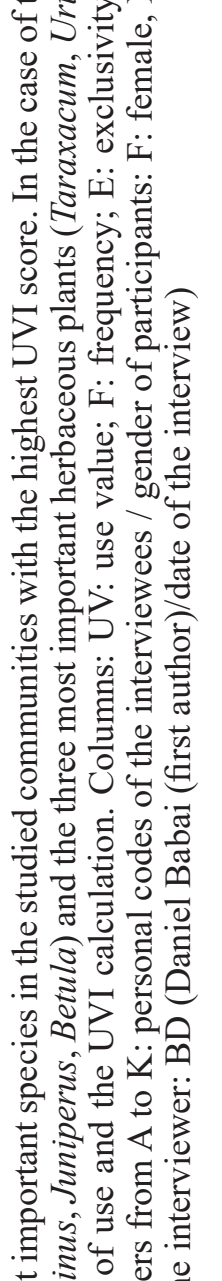 & 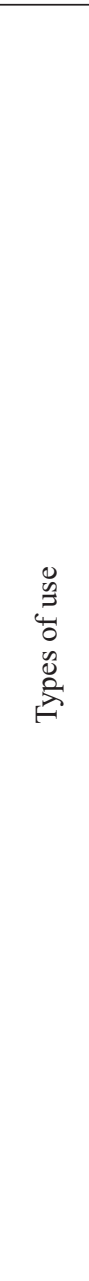 & 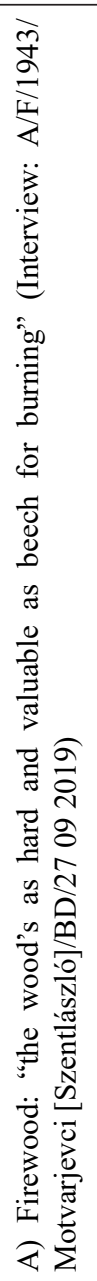 & 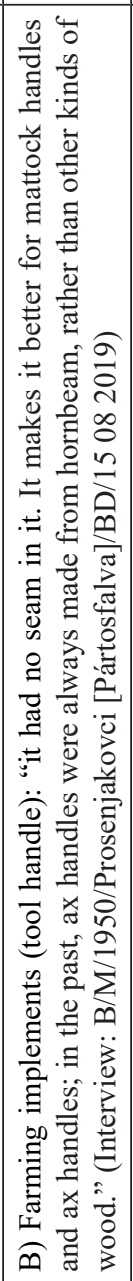 & 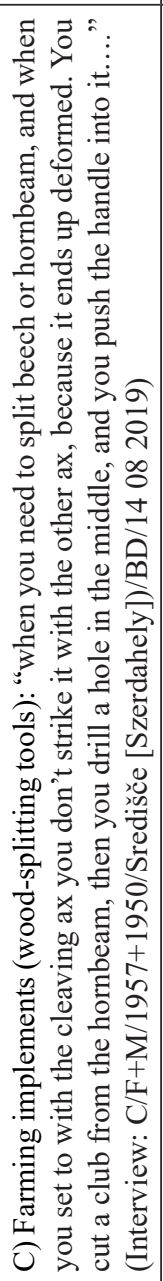 & 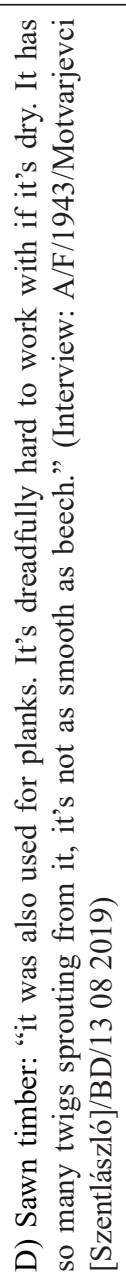 & हैं \\
\hline 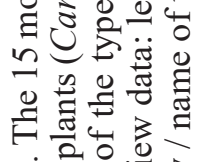 & 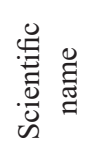 & & & 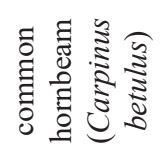 & & \\
\hline 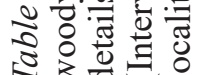 & $\bar{z}$ & & & & & \\
\hline
\end{tabular}


Dániel Babai et al.

\begin{tabular}{|c|c|c|c|c|c|c|}
\hline 3 & 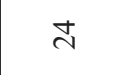 & $\underset{\sim}{\stackrel{J}{ }}$ & $\stackrel{\beth}{\sim}$ & 0 & $m$ & $\bar{\infty}$ \\
\hline 피 & $N$ & N & $\sim$ & - & - & \\
\hline 山 & $\nabla$ & $\nabla$ & $\nabla$ & $\sim$ & - & \\
\hline 3 & $m$ & $m$ & $m$ & $m$ & $m$ & \\
\hline 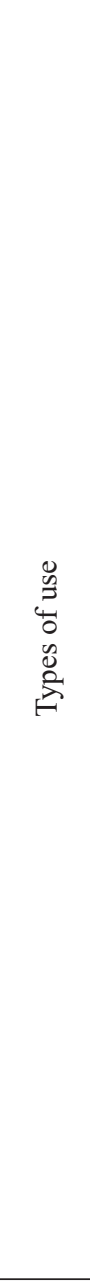 & 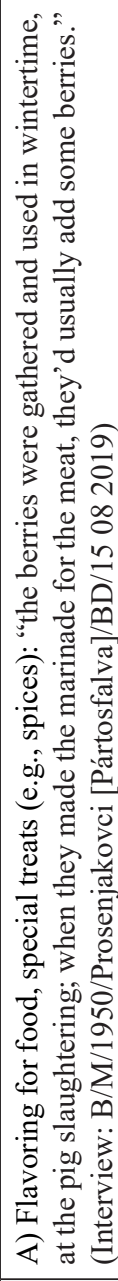 & 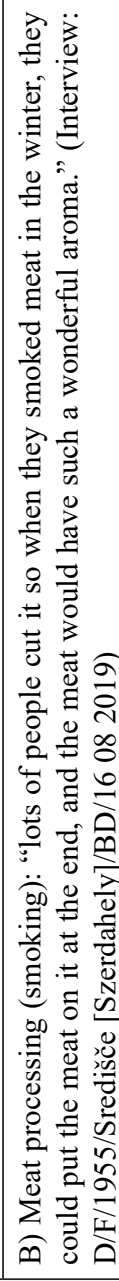 & 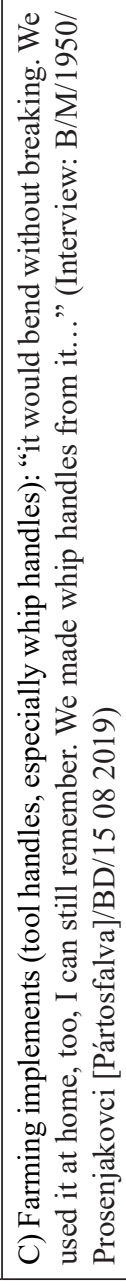 & 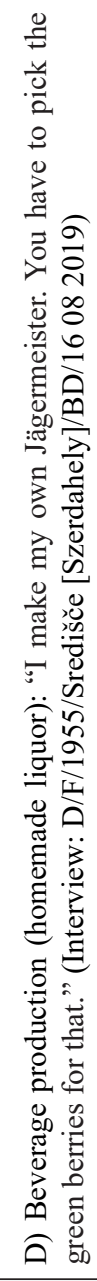 & 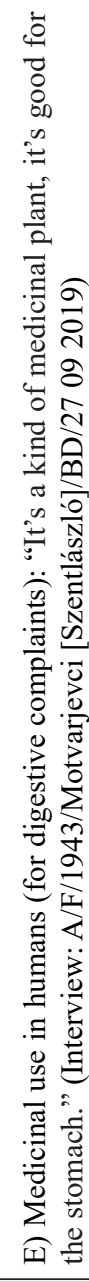 & हैّ \\
\hline 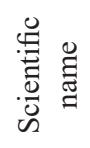 & \multicolumn{6}{|c|}{ 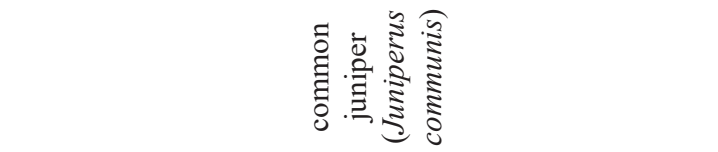 } \\
\hline z & \\
\hline
\end{tabular}


Traditional Ecological Knowledge and the Cultural Significance of Plants... 499

\begin{tabular}{|c|c|c|c|c|c|c|c|c|c|c|}
\hline 5 & ల & $\infty$ & $\infty$ & $\infty$ & $\infty$ & $\nabla$ & $\nabla$ & $m$ & m & $\stackrel{2}{2}$ \\
\hline س & $N$ & $N$ & - & $N$ & $N$ & - & - & - & - & \\
\hline 山 & in & - & 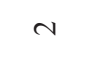 & - & $N$ & 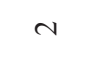 & $N$ & - & - & \\
\hline 3 & $n$ & $\nabla$ & $\nabla$ & ナ & $N$ & 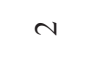 & $N$ & $m$ & $m$ & \\
\hline \begin{tabular}{l}
0 \\
0 \\
0 \\
0 \\
0 \\
0 \\
0 \\
\multirow{0}{*}{}
\end{tabular} & 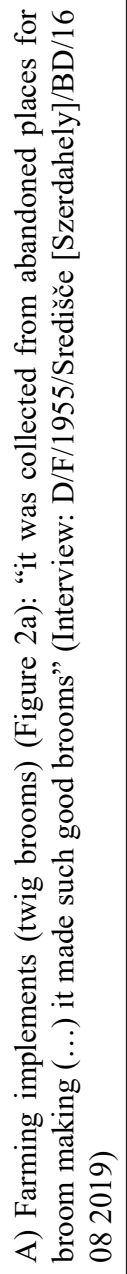 & 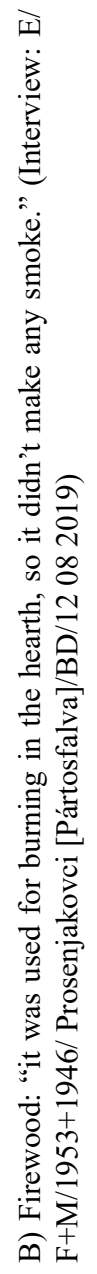 & 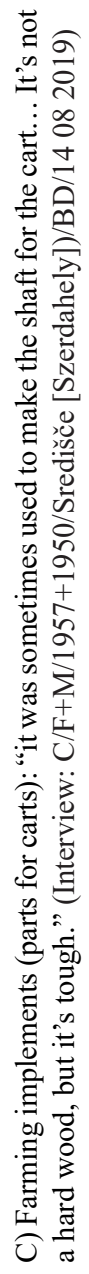 & 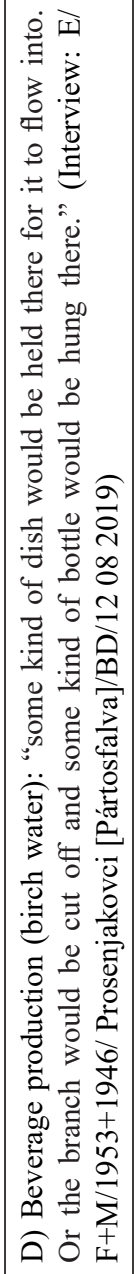 & 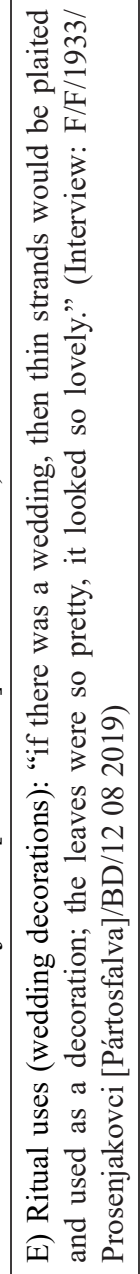 & 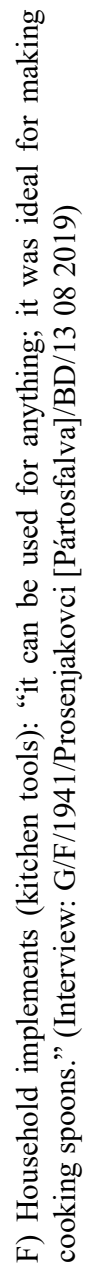 & 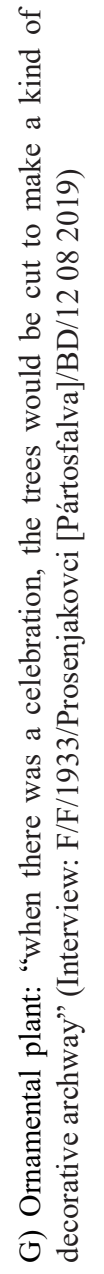 & 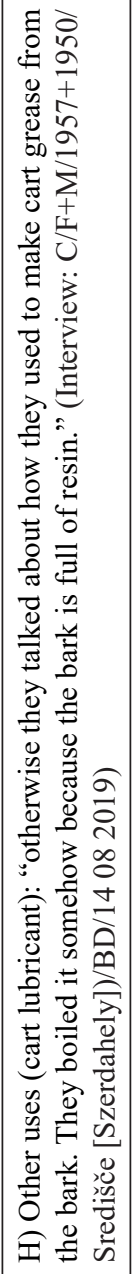 & 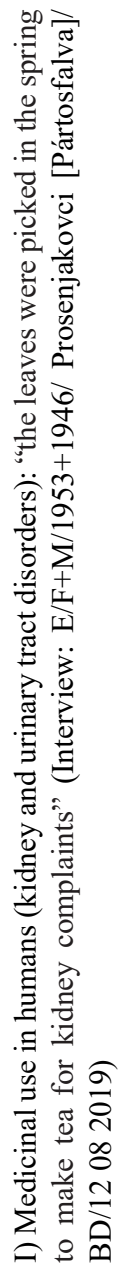 & ब⿳亠二厶凵 \\
\hline 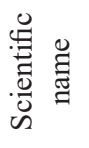 & \multicolumn{10}{|c|}{ 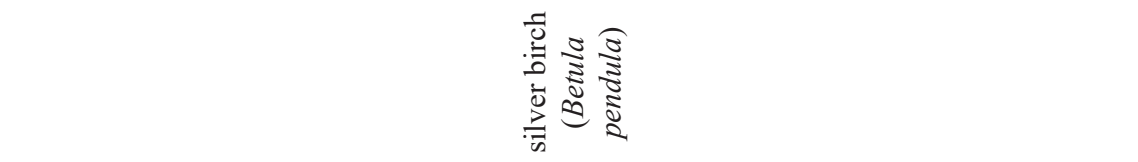 } \\
\hline $\bar{z}$ & \multicolumn{10}{|c|}{$m$} \\
\hline
\end{tabular}


Dániel Babai et al.

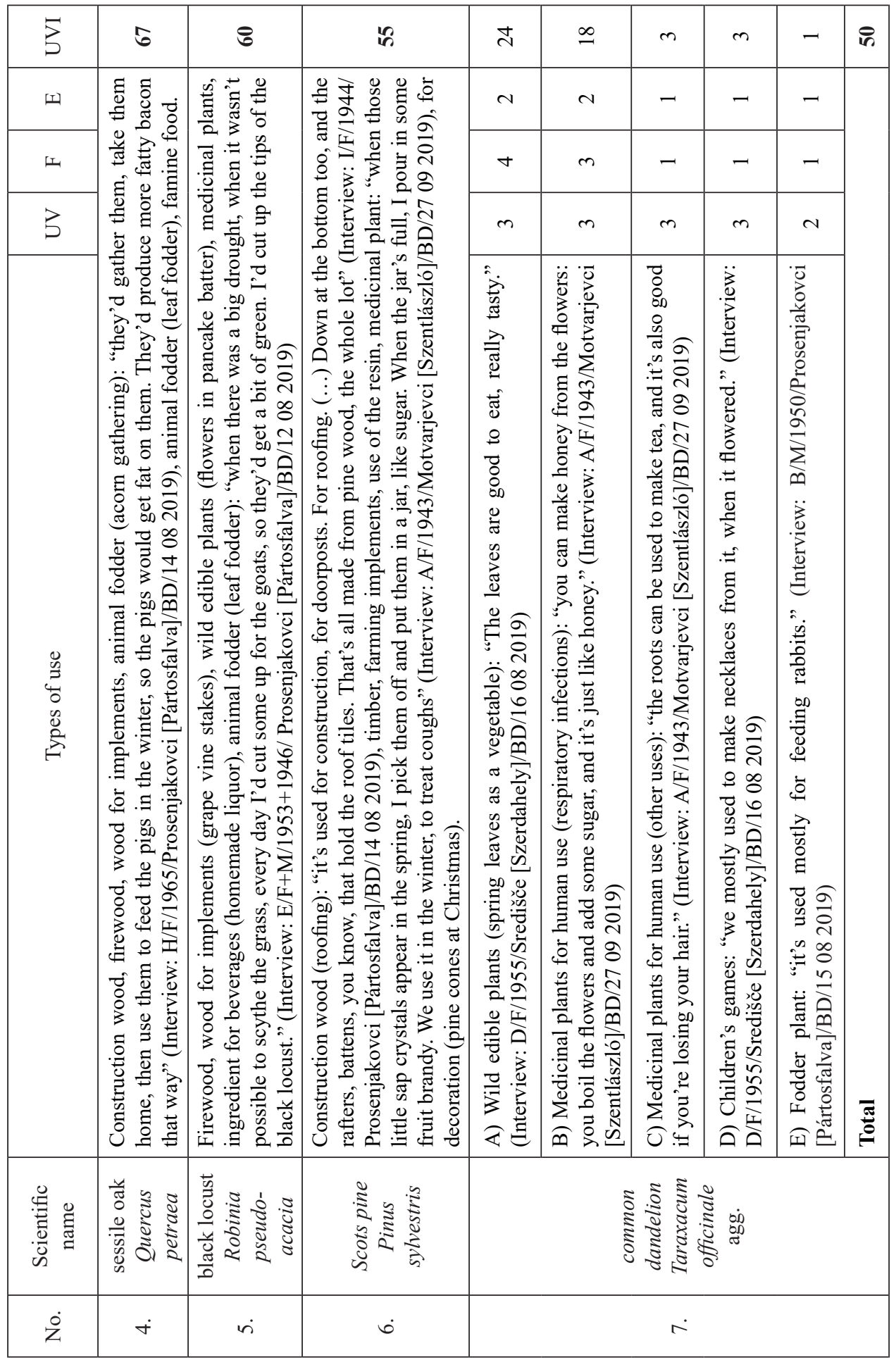


Traditional Ecological Knowledge and the Cultural Significance of Plants... 501

\begin{tabular}{|c|c|c|c|c|c|c|c|c|c|}
\hline 5 & $\stackrel{\infty}{\sim}$ & $\simeq$ & $a$ & $m$ & $m$ & 4 & 7 & $\bar{F}$ & 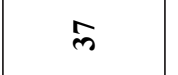 \\
\hline 디 & $N$ & $d$ & 4 & - & & & \multirow[b]{4}{*}{ 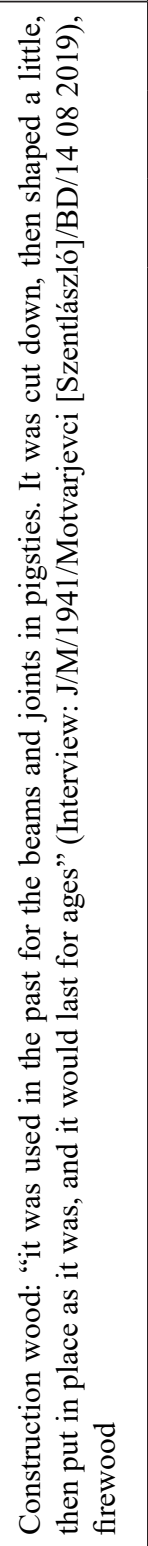 } & \multirow[b]{4}{*}{ 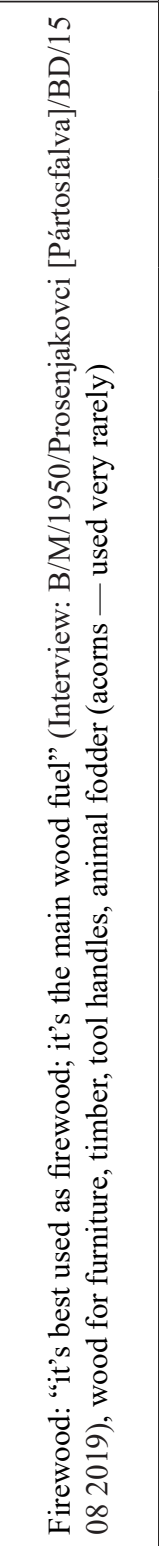 } & \multirow[b]{4}{*}{ 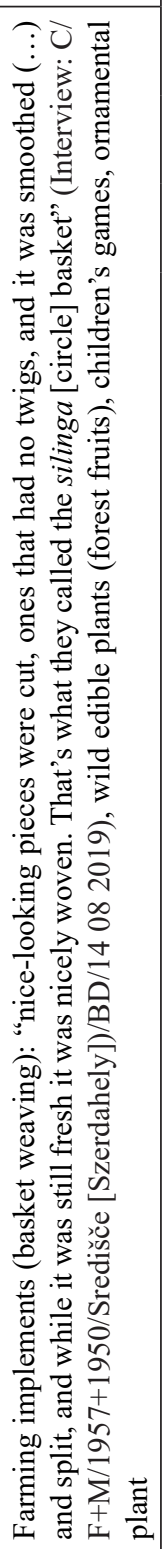 } \\
\hline L & $m$ & $N$ & $m$ & - & - & & & & \\
\hline 3 & $m$ & $n$ & $m$ & $m$ & & & & & \\
\hline \begin{tabular}{l}
0 \\
0 \\
0 \\
0 \\
0 \\
0 \\
0 \\
\multirow{2}{*}{}
\end{tabular} & 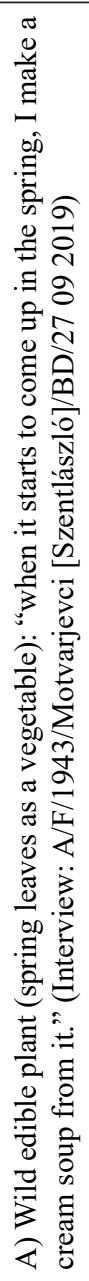 & 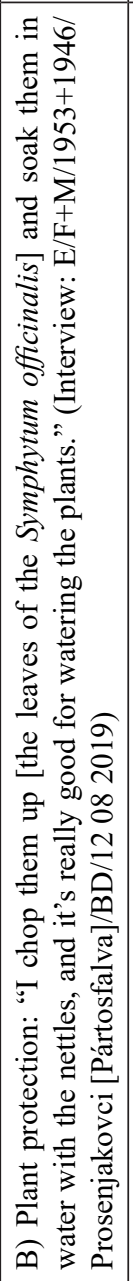 & 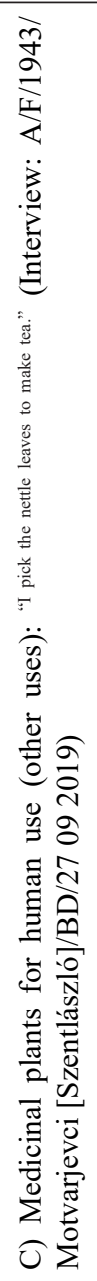 & 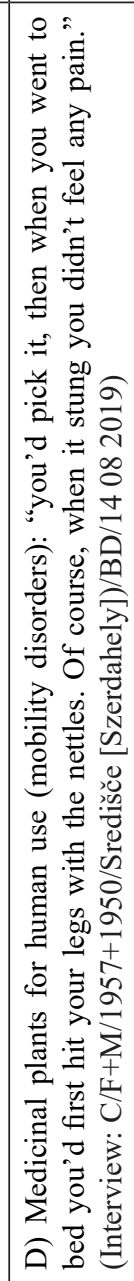 & 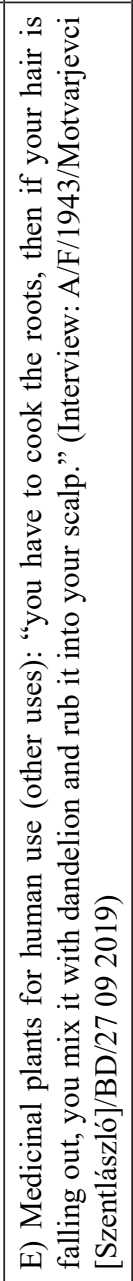 & 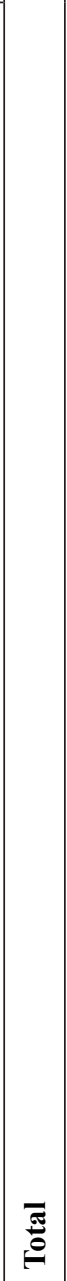 & & & \\
\hline 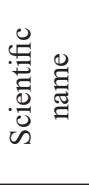 & \multicolumn{6}{|c|}{ 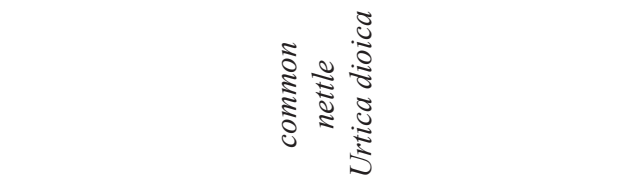 } & 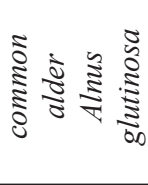 & 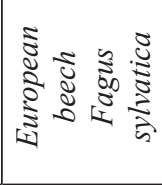 & రి \\
\hline$z$ & \multicolumn{6}{|c|}{$0^{\circ}$} & & & \\
\hline
\end{tabular}


Dániel Babai et al.

\begin{tabular}{|c|c|c|c|c|c|c|c|c|}
\hline 5 & t & $\mathbb{V}$ & ల & 6 & लि & $\vec{n}$ & & 5 \\
\hline 되 & $N$ & $N$ & & \multirow[b]{4}{*}{ 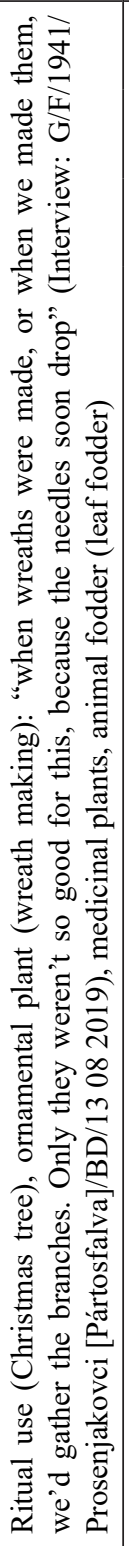 } & \multirow[b]{4}{*}{ 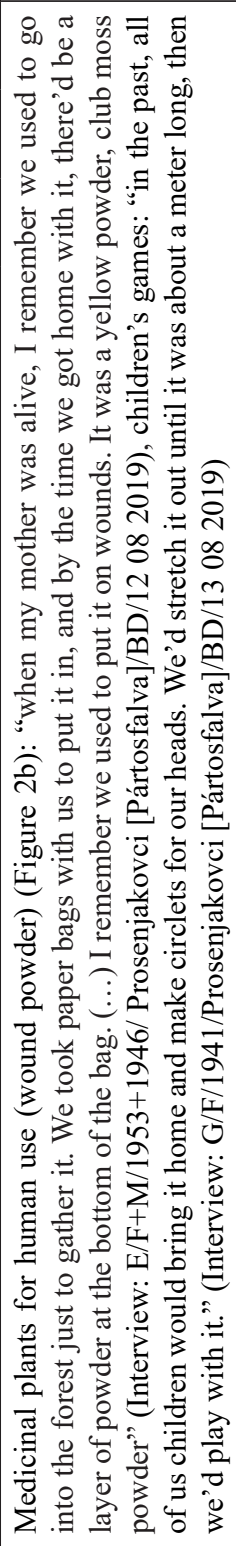 } & \multirow[b]{4}{*}{ 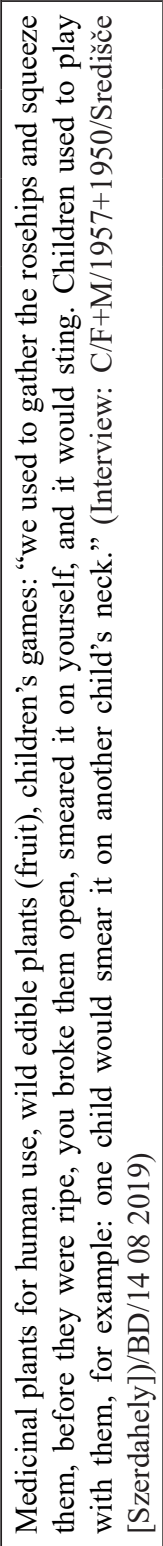 } & \multirow[b]{4}{*}{ 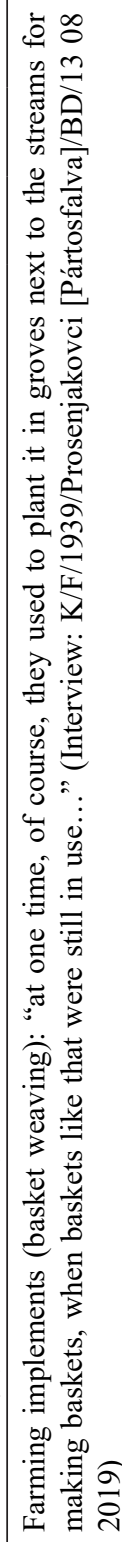 } & \multirow[b]{4}{*}{ 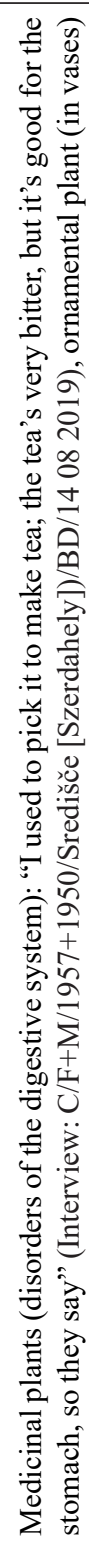 } \\
\hline 山 & $\nabla$ & $m$ & & & & & & \\
\hline 3 & $m$ & 0 & & & & & & \\
\hline 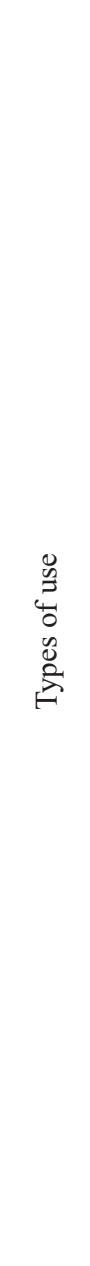 & 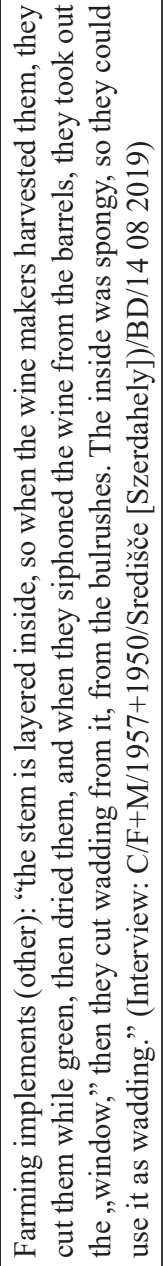 & 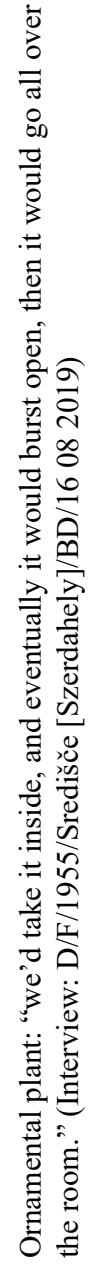 & 产 & & & & & \\
\hline 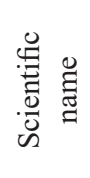 & \multicolumn{3}{|c|}{ 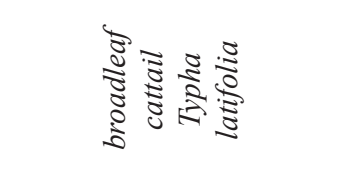 } & 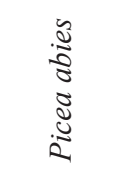 & 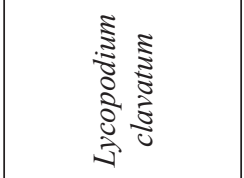 & $\begin{array}{l}\text { : } \\
\text { ह } \\
0 \\
0\end{array}$ & 离 & 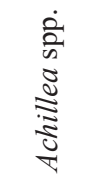 \\
\hline$z$ & & & & \\
\hline
\end{tabular}




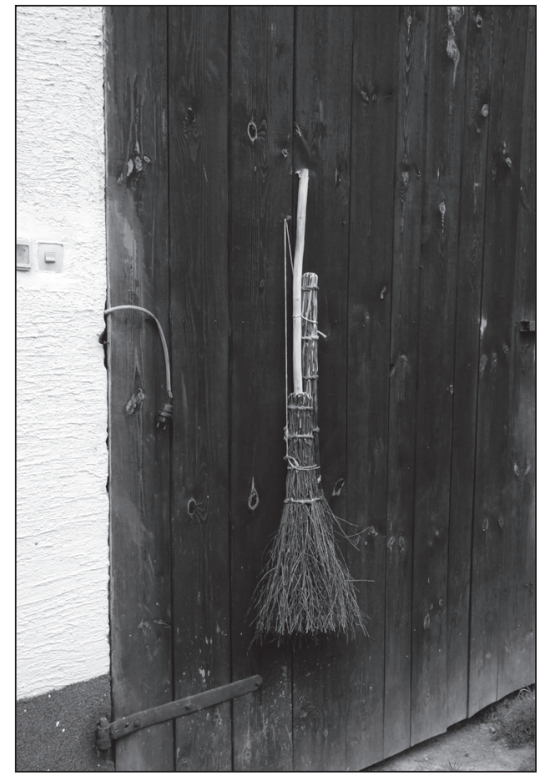

Figure 2a. Broom made from birch (Betula pendula), Motvarjevci (Szentlászló), Slovenia, 2019. (Photo by Dániel Babai)

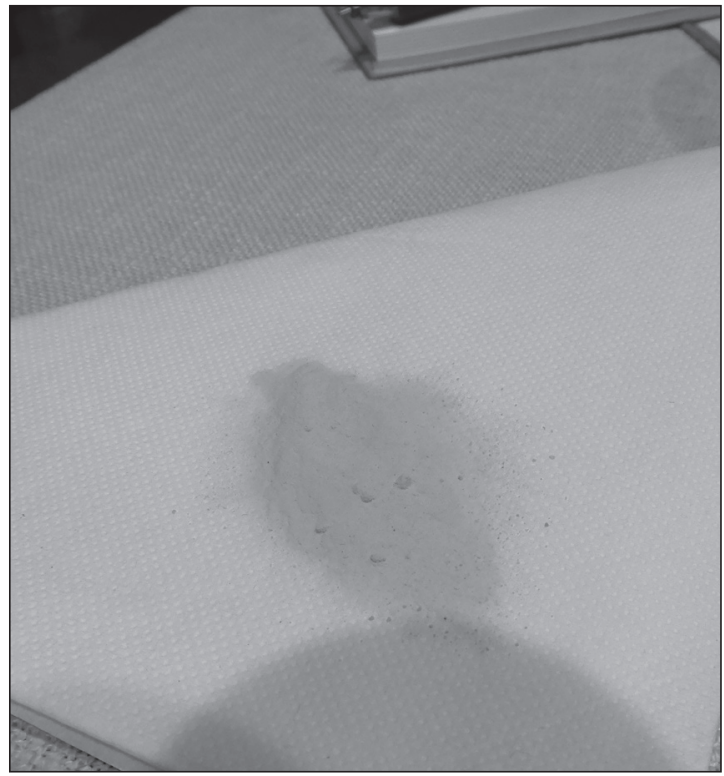

Figure 2b. Spores of the club-moss (Lycopodium clavatum), which were once gathered in large quantities for use as wound powder. Središče (Szerdahely), Slovenia, 2018. (Photo by Viktor Ulicsni)

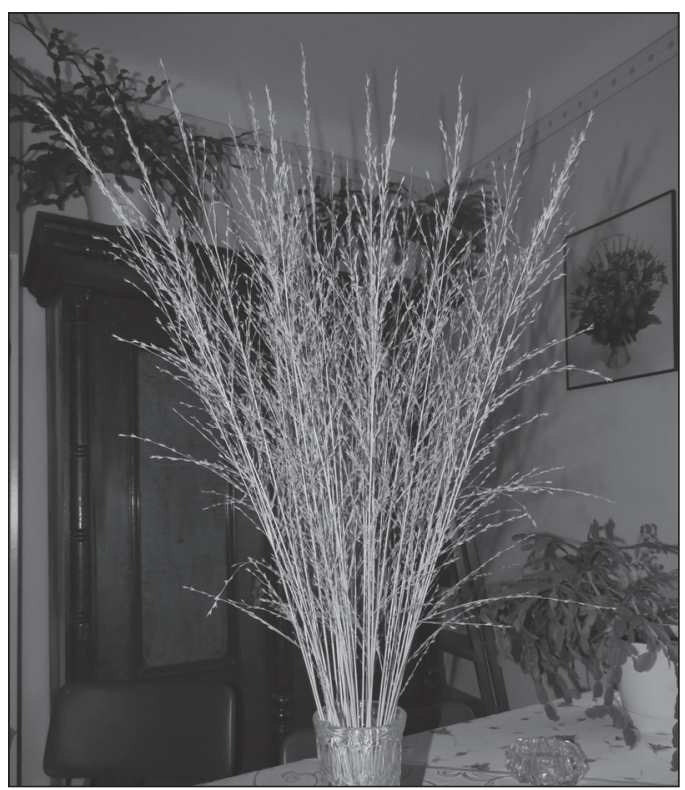

Figure 2c. Molinia caerulea used as dried flowers in a home. Prosenjakovci (Pártosfalva), Slovenia, 2019. (Photo by Dániel Babai) 


\section{DISCUSSION}

\section{Characteristics of traditional ecological knowledge of plants}

In the studied region, the number of folk taxa identified to date is relatively low, compared to the flora as a whole (see MolNÁR - BABAI 2009; MolnÁR 2011; PÉNTEK - SZABÓ 1985). The reason for this may be methodological (we did not ask about every single species), or it may be due to the erosion of plant knowledge (having conducted interviews with 20 interviewees, it appears that the species knowledge curve is becoming saturated: no new local names were mentioned in response to repeated questions). Based on the interviews, as well as the known ethnobotanical data from the wider region (BAZSIKA 2010; BöDEI 1943; KARDOs 1943; TóTH 2009), we estimate that between 80 and $85 \%$ of the folk taxa that are still preserved in living memory may have been mentioned.

Forest vegetation was well known - both the vast majority of tree species that form a canopy layer (interestingly, with the exception of maple species, which were known in Göcsej: BAZSIKA 2010; BöDEI 1943); and species belonging to the shrub layer. Information related to several shrub species was not (yet?) mentioned: species of Euonymus and Cornus sanguineus (known in Göcsej: BAZsIKA 2010; BöDEI 1943). Among the species of the herbaceous layer, Lycopodium clavatum was well known. Members of the Slovenian population dried and ground not only the spores, but also the above-ground parts of the plant, and used them as wound powder (MLAKAR 2015:89, 364). Most of the interviewees mentioned the dwindling of this species. Both overexploitation and changes in land use were mentioned as reasons for its disappearance. In the botanical literature, the discontinuation of forest litter collection and the resulting changes in the forest soils are primarily blamed for the drastic decrease in the populations of the species (GRUBER et al. 2015:15).

The flora of hay meadows was less well known compared to the plant knowledge of smallholders in Ghimeș (Gyimes), Romania, who are engaged in similar agricultural activities (animal husbandry, hay cultivation) (cf. BABAI et al. 2014; BABAI - MoLnÁR 2016; MolnáR - BABAI 2009). The dominant, narrow-leaved species of top grass were not known on species level in either region (in terms of hay making, there is no practical importance in differentiating between species that are ecologically similar). In Ghimeș, the various different top grasses (around 21 species) are classified under one taxon (species group) under the name of imola (BABAI et al. 2014; BABAI - MoLNÁR 2016). In the studied villages, the taxon of top grasses has a separate name: füjj. Only one species is remarkable from a perceptual point of view — quaking grass (Briza media), which has been given a local name in both regions (poloskafü [bug grass] in Ghimeș, and Máriakönnye [Mary's tears]) in the study area, and is given a distinct name in Trei Scaune (Háromszék) and Gheorgheni (Gyergyó) as well (cf. BABAI et al. 2014; MolnáR - BABAI 2009; PÉNTEK - SzABÓ 1976; RAB 2001:208). It was used in Göcsej as a medicinal plant, as well as for predicting a woman's future husband (BöDEI 1943:81), while in Gheorgheni it was also used as a dried flower ( $\mathrm{RAB} 2001: 125,208)$. Among the striking flowered species growing in hay meadows, the ragged robin (Lychnis flos-cuculi) and the green-winged orchid (Anacamptis morio) were well known. The name "sárga virág" [yellow flower] for the meadow buttercup (Ranunculus acris), which typically grows in large numbers, seems to be fairly consistent (not merely as an adjectival construction), and is an indicator 
species forecasting the quantity of hay. Almost everyone was familiar with the great burnet (Sanguisorba officinalis), a highly valued fodder plant in the Örség region, a conspicuous plant that grows in large quantities at the end of the summer, at the time of the second mowing, although it has no name (there was only one mention of a name - birkabogározó füjj - which was not confirmed by any of the other interview subjects).

The weeds associated with arable lands and kitchen gardens were well known. Some of the species (corncockle: Agrostemma githago; cornflower: Centaurea cyanus; common poppy: Papaver rhoeas) were used as ornamental plants (in vases). These species were well known, despite the fact that, because of the intensification of agriculture and the increased use of herbicides, they disappeared almost entirely from arable land decades ago (

\section{Culturally salient species}

Interviewees listed the utilization of the 130 folk taxa in a great variety of ways. A significant proportion of the uses are similar to data published in other regions of the Carpathian Basin. The cultural keystone species, as in other, essentially agricultural communities that pursue wild plant gathering merely as a complementary activity, are primarily woody plants, due to the importance of the utilization of timber (PÉNTEK SZABÓ 1985:159-160; RAB 2001:123-124). The wood of the common hornbeam, silver birch, beech, or oak played a very important role in tool making, as firewood, and as construction materials, etc. in the daily lives of households and farms. With respect to the common juniper (Juniperus communis), there were several exclusive uses, where juniper could not be substituted by other species (e.g. the use of Juniper berries as a spice for marinating meats, and the use of the wood for smoking meat or for making whip handles). Its use as a spice and as a medicinal plant was also typical among the Slovenian population (MLAKAR 2015). The silver birch (Betula pendula) was utilized with remarkable diversity, having as many as six different uses earlier. Among the farming implements, the making of twig brooms remains important even today. The diversity of its use was also typical of other regions in the Carpathian Basin, such as Transylvania (cf. PAPP et al. 2014b; PÉNTeK - SzABó 1985:209; TARISZnYás 1978:30-31), while the Slovenians primarily used its sap (MLAKAR 2015; NovaK 1957). The role played by tree species in medicine and nutrition (e.g. the consumption of the fresh, spring leaves) may have been smaller in the Hungarian communities in Slovenia than in other regions of the Carpathian Basin (DÉNES et al. 2012: 384; PÉNTEK - SzABÓ 1985:112).

The herbaceous plants that played the most important role in the everyday life of the Hungarian community in Slovenia - the common dandelion (Taraxacum officinale agg.) and the common nettle (Urtica dioica) - were species that were also frequently and diversely used in other regions in the Carpathian Basin (e.g. wild edible plants, medicinal plants, fiber crops) (e.g. GrYnaeus - Szabó 2002:211; Halász 2010), and were also used by the neighboring Slovenian population (MAKAROVIČ 1988; MLAKAR 2015; PAPEŽ 2010). The importance of the broadleaf cattail (or bulrush: Typha latifolia) had increased due to the utilization of its spongy, insulating properties (as in Göcsej - BAZsiKa 2010). In other regions, it was mentioned for its role in the weaving of household implements, as famine food, or in extensive pig rearing (BENCSIK 1973). 
The number of species used as ornamental plants was extremely high. The methods used to create arrangements from forget-me-not species (Myosotis spp.) are especially interesting for their complexity. The number of medicinal and wild edible plant species is also high (39 in each case) (cf. BABAI et al. 2014; PAPP - HoRváth 2013; PÉNTEK - SzABó 1976, 1985; RAB 2001). The use of medicinal plants that are still considered important today in the living memory (e.g. species of yarrow [Achillea spp.]; common centaury [Centaurium erythreae]; and stag's-horn clubmoss [Lycopodium clavatum]) is similar to the official methods of application in the case of Achillea (digestive complaints), Centaurium (appetite stimulant), and Betula (kidney complaints) (cf. DÉNES et al. 2014). Besides these species, in Kardos's Örség collection (1943: 82), linden (Tilia sp.), carroway (Carum carvi), and elder (Sambucus nigra) are also highly important medicinal plants. However, we found only sporadic information concerning the medicinal use of these species. On the basis of the accessible regional scientific literature there is no significant change in terms of species of wild edible plants. Among the edible species of the folk taxa that we also explored, Kardos (1943:9-10) mentions in his research in the Örség region primarily forest fruits (epörgye - wild strawberry [Fragaria vesca]; málna - raspberry [Rubus idaeus]; tüskeszeder - blackberry [Rubus fruticosus]; seggibugya [called csipkënye by Kardos] rosehip [Rosa canina agg.]; gereginye-tüske [called kökin by Kardos] - blackthorn [Prunus spinosa]; vadalma - crabapple [Malus sylvestris]; vadkörte - wild pear [Pyrus pyraster]; and magyaru - common hazel [Corylus avellana]). The use of the bird cherry (Prunus padus) and common juniper (Juniperus communis) for making fruit brandy (pálinka) was not mentioned (cf. KARDOS 1943:10). The single-seeded hawthorn (Crataegus monogyna) was not widely known in the Hungarian settlements in Slovenia. At the same time, according to Kardos's research (1943:10), the red berry of the Crataegus (galagonya) was known by the name geleginye in settlements in the Örség region, while Bazsika (2010) mentions it by the name of geregënye in Göcsej. In the Hungarian communities in Slovenia, this name (in the form gereginye) refers unambiguously and consistently to the blackthorn (kökény [Prunus spinosa]) (while the name kökény is also known), often with a reference to the color: the gereginye has black or dark blue berries. It's very sour: it's only good if it's been slightly frozen. ${ }^{4}$

Since a significant proportion of the members of those generations that still possess traditional ecological knowledge no longer have any direct and active connection with the natural environment, it follows that knowledge of plant species, local names, and often the precise ways in which species were once used have been lost from memory. Extensive land use, which György Nemesnépi Zakál referred to in his work on the Örség region, is now a thing of the past: "I have often observed these 'Students of Nature,' who gather seeds, grasses, and roots" (quoted in KARDOS 1943:10). The significance of this lies in the fact that in folk plant knowledge, the examination and observation that take place in the course of practical use give rise to sound species knowledge (among the 122 named taxa found in our documentation, in only 11 cases no information concerning practical use was mentioned) (PÉNTEK - SzABÓ 1976:222).

4 Interviewee D (woman, 1955, Središče [Szerdahely]). Interview was conducted by Daniel Babai (16 08 2019). 


\section{CLOSING THOUGHTS}

While knowledge of nature is fading in human communities with the disappearance of experience and abandonment of land use, the vegetation and the species themselves are changing with changes in land use, and the region's biodiversity is being restructured. However, there are some very knowledgeable people who are willing to help us document that segment of Hungarian folk culture that is becoming a thing of the past, and to contribute to an understanding of our connections with land use and vegetation, preserving the memory of earlier relationships between the landscape and the human population.

\section{ACKNOWLEDGEMENTS}

The authors would like to express their gratitude to all of the interviewees, whose enthusiastic help was an enormous support to us during the research, and all of whom recognized the importance of documenting and preserving the memories of this knowledge. The authors express their special thanks to Dr. Dragica Purger for helping to process Slovenian ethnobotanical literature.

The work of Dániel Babai was supported by a Premium Postdoctoral Fellowship (PPD 008/2017) from the Hungarian Academy of Sciences, and the work of Viktor Ulicsni was supported by the National Research, Development, and Innovation Office research project "Protected Areas along the Slovenian-Hungarian Border: Challenges of Cooperation and Sustainable Development" (SNN 126230).

\section{REFERENCES CITED}

BABAI, Dániel - Molnár, Ábel - Molnár, Zsolt

2014 „Ahogy gondozza, úgy veszi hasznát” Hagyományos ökológiai tudás és gazdálkodás Gyimesben [Traditional Ecological Knowledge and Land Use in Gyimes (Eastern Carpathians, Romania)]. Budapest-Vácrátót: MTA Research Centre for the Humanities, Institute of Ethnology and MTA Centre for Ecological Research, Institute of Ecology and Botany.

BABAI, Dániel - MoLnár, Zsolt

2014 Small-scale traditional management of highly species-rich grasslands in the Carpathians. Agriculture, Ecosystem and Environment 182:123-130.

2016 Species-rich Mountain Grasslands Through the Eyes of the Farmer: Flora, Species Composition, and Extensive Grassland Management. Martor. A Place BAZsIKA, Enikö

for Hay. Flexibility and Continuity in Hay-Meadow Management 21:147-169.

2010 Népi növény- és állatnevek Göcsejben [Folk plant and animal names in Göcsej]. Muratáj Lendva - Lendava 2009(1-2):77-96. 
BENCSIK, János

1973 A gyüjtögető gazdálkodás emlékei a Tisza mentén, a volt alsó-szabolcsi falvakban [Memories of food-gathering along the Tisza river, in the villages of the former Lower Szabolcs region]. Hajdúsági Múzeum Évkönyve 1:111-126.

BERKES, Fikret

2012 Sacred ecology. New York: Routledge.

BöDEI, János

1943 Adatok Zalabaksa gyüjtögető gazdálkodásához [Data on food-gathering in Zalabaksa]. Néprajzi Értesítö 35:69-96.

Cousins, Sara A. O. - Kaligarič, Mitja - BAKAn, Branko - Lindborg, Regina

2014 Political Systems Affect Mobile and Sessile Species Diversity - A Legacy from the Post-WWII Period. Plos ONE 9(8):e103367. doi:10.1371/journal. pone. 0103367

DÉnEs, Andrea - PAPP, Nóra - BABAI, Dániel - Czúcz, Bálint - MolnÁR, Zsolt

2012 Wild plants used for food by Hungarian ethnic groups living in the Carpathian Basin. Acta Societatis Botanicorum Poloniae 81:381-396.

DÉnes, Tünde - TóTH, Mónika - GYergyák, Kinga - LöRInCz, Péter - VARGA, Erzsébet

- PAPp, Nóra

2014 Szemelvények Homoródalmás (Erdély) népi gyógynövényismeretéből [Ethnobotanical data from Homoródalmás (Meresti, Romania)]. Botanikai Közlemények 101:227-241.

von Glasenapp, Markus - ThORnTOn, Thomas F.

2011 Traditional ecological knowledge of Swiss Alpine farmers and their resilience to socioecological change. Human Ecology 39:769-781.

Gosler, Andrew G.

2017 The Human Factor: Ecological Salience in Ornithology and Ethno-ornithology. Journal of Ethnobiology 37:637-662.

Gruber, Ágnes - HarsánYI, Krisztián - Havas, Márta - Horváth, Jenő - Kevy, Albert

- Kovács-Mesterházy, Zoltán - Markovics, Tibor - Németh, Csaba - PÁL, Éva SzEnTIRMAi, István - SzÉPligeti, Mátyás - TANNER, Tibor - TÁRCZY, Andrea

2015 Jelentés az Örségi Nemzeti Park Igazgatóság 2014. évi tevékenységéröl [Report on the Activities of the Örség National Park Directorate in 2014]. Öriszentpéter: Örségi Nemzeti Park Igazgatóság.

GrYNAEUS, Tamás - SzABó, László Gy.

1993 Növények ismerete és használata Dávodon (Bács-Kiskun m.) [Knowledge and Use of Plants in Dávod, Bács-Kiskun County]. Gyógyszerészet 37:29-36., 85-92.

2002 A bukovinai hadikfalvi székelyek növényei (növénynevek, növényismeret és -felhasználás) [Plants of the Hadikfalva Székelys of Bukovina (Plant Names, Plant Knowledge and Plant Use)]. Kriza János Néprajzi Társaság Évkönyve 10:153-246.

Halász, Péter

2010 Növények a moldvai magyarok hagyományában és mindennapjaiban [Plants in the tradition and everyday life of the Hungarians in Moldova]. Budapest: General Press. 
HunN, Eugene, S.

1999 Size as Limiting the Recognition of Biodiversity in Folkbiological Classifications: One of Four Factors Governing the Cultural Recognition of Biological Taxa. In Medin, Douglas. L. - Atran, Scott (eds.) Folkbiology, 47-69. Cambridge: Harvard University Press.

Kaligarič, Mitja - SEDONJA, Jožef - ŠAJnA, Nina

2008 Traditional Agricultural Landscape in Goričko Landscape Park (Slovenia): Distribution and Variety of Riparian Stream Corridors and Patches. Landscape and Urban Planning 85:71-78.

KARDOS, László

1943 Az Örség népi táplálkozása [Folk Nutrition in the Örség Region]. Budapest: Államtudományi Intézet Táj- és Népkutató Osztálya.

KIRÁLY, Gergely (ed.)

2009 Új magyar füvészkönyv. Magyarország hajtásos növényei. Határozókulcsok. [New Hungarian Herbal. The Vascular Plants of Hungary. Identification Key]. Jósvafő: Aggteleki Nemzeti Park Igazgatóság.

KLADNIK, D. - REPOLUSK, P.

1993 Arurális térségek fejlődésének szerepe a magyar etnikai identitás megőrzésében az alsólendvai kommunában (Szlovénia) [The Role of the Development of Rural Areas in the Preservation of the Hungarian Ethnic Identity in the Commune of Alsólevendva]. Földrajzi Közlemények 117:235-250.

Kóczián, Géza

1984 Etnobotanikai vizsgálatok Répáshután [Ethnobotanical Studies in Répáshuta]. In Szabadfalvi, József - Viga, Gyula (eds.) Répáshuta. Egy szlovák falu a Bükkben, 229-256. Miskolc: Herman Ottó Múzeum Néprajzi Kiadványa, XIII.

1988 A taranyi cigányok gyógyító és mérgező növényei [Medicinal and Poisonous Plants of the Roma of Tarany]. Gyógyszerészet 32:417-421.

2014 A hagyományos parasztgazdálkodás termesztett, a gyüjtögető gazdálkodás vad növényfajainak etnobotanikai értékelése [An Ethnobotanical Assessment of Wild Plant Species Cultivated in Traditional Peasant Farming and in FoodGathering]. Nagyatád: Nagyatádi Kulturális és Sport Központ, Nagyatád.

Kóczián, Géza - Szabó, László Gy.

1990 A szlovákiai Áj és Falucska községek népeinek gyógynövényhasználata, etnobotanikai tudása. Adatközlés [The Use of Medicinal Plants and the Ethnobotanical Knowledge of People Living in the Slovakian Villages of Háj (Áj) and Hačava (Falucska)]. Gyógyszerészet 34:371-377.

MAKAROVIČ, Gorazd

1988/90 Prehrana v 19. stoletju na Slovenskem. [Diet in Slovenia in the $19^{\text {th }}$ Century]. Slovenski Etnograf 33/34:127-205.

MLAKAR, Vlasta

2015 Rastlina je sveta, od korenin do cveta: tradicionalno znanje o rastlinskem svetu na Slovenskem. [Traditional Knowledge on Plants in Slovenia]. Ljubljana: self publishing.

Molnár, Zsolt

2011 A Hortobágyi pásztorok növényosztályozása, a vadon termő növények ismertsége és néven nevezettsége [Plant Classification, Knowledge of Wild 
Plants, and Denoting by Name among the Herdsmen of Hortobágy]. Crisicum 7:153-207.

Molnár, Zsolt - BABAI, Dániel

2009 Népi növényzetismeret Gyimesben I: Növénynevek, népi taxonómia, az egyéni és közösségi növényismeret [Folk Plant Knowledge in Gyimes I.: Plant Names, Folk Taxonomy, Plant Knowledge on Individual and Community Level]. Botanikai Közlemények 96:117-143.

Móra, Ferenc

1960 Népies növények a Kiskunság flórájában [Popular Plants in the Flora of the Kiskunság Region]. In VAJdA, László - MADÁcsy, László (eds.) A fele sem tudomány. Utazás a földalatti Magyarországon, 254-261. Budapest: Magvető.

Newing, Helen S.

2010 Conducting Research in Conservation. A social science perspective. LondonNew York: Routledge Taylor \& Francis Group.

NovaK, Vilko

1957 Vprašanje nabiralništva pri Slovencih [Question about Gathering of Wild Plants by Slovenian People]. Slovenski Etnograf 10:19-28.

PAPEŽ, Andreja

2010 Uporaba divje rastočih rastlin v prehrani [Use of Wild-Growing Edible Plants in Human Nutrition]. Graduate thesis, Ljubljana: University of Ljubljana.

Papp, Nóra - Birkás-Frendl, Kata - FArkas, Ágnes - CzéGÉnYi, Dóra

2014a Hungarian Ethnobotanical Studies in Romania. In PIERONI, Andrea - QuAve, Cassandra (eds.) Ethnobotany and Biocultural Diversities in the Balkans: Perspectives on Sustainable Rural Development and Reconciliation, 29-44. New York: Springer Verlag.

Papp, Nóra - Czégényi, Dóra - Hegedüs, Anita - MorschHauser, Tamás - Quave, Cassandra L. - Cianfaglione, Kevin - Pieroni, Andrea

2014b The uses of Betula pendula Roth among Hungarian Csángós and Székelys in Transylvania, Romania. Acta Societatis Botanicorum Poloniae, 83:113-122.

PAPP, Nóra - HoRváTH, Dávid

2013 Vadon termő ehető növények Homoródkarácsonyfalván (Erdély) [Wild Edible Plants in Crăciunel (Transylvania)]. In DÉNES, Andrea (ed.) Ehetö vadnövények a Kárpát-medencében, 83-92. Pécs: Janus Pannonius Múzeum.

PAUŠIČ, Igor - IVAJNŠIČ, Danijel - KAIGARIČ, Mitja - PIPENBAHER, Nataša

2017 Relation between plant species diversity and landscape variables in CentralEuropean dry grassland fragments and their successional derivates. Acta Botanica Croatica 76:111-119.

PÉNTEK, János - SzABó, T. Attila

1976 Egy háromszéki falu népi növényismerete [Ethnobotanical Studies in a Village in Trei Scaune]. Ethnographia 87:203-225.

1985 Ember és növényvilág. Kalotaszeg növényzete és népi növényismerete [People and Plantlife. The Vegetation and Folk Plant Knowledge of Kalotaszeg]. Bukarest: Kriterion.

RAB, János

2001 Népi növényismeret a Gyergyó-medencében [Folk Plant Knowledge in the Gyergyó Basin]. Csíkszereda: Pallas - Akadémiai. 
ŠILC, Urban - ČARNI, Andraž

2005 Changes in weed vegetation on extensively managed fields of central Slovenia between 1939 and 2002. Biologia, Bratislava 60:1-8.

SzÜCs, Sándor

2003 A gyékény földolgozása és eszközei a Biharmegyei Sárréten [The processing of bulrushes and the related tools in the Sárrét area of Bihor (Bihar) County]. In Dankó, Imre (ed.) Szücs Sándor. A puszta utolsó krónikása, 15-131. Túrkeve: Finta Múzeumért Alapítvány.

TARISZNYÁs, Márton

1978 A gyüjtögető gazdálkodás hagyományai Gyergyóban [Traditions of the Gatherer Agriculture in Gheorgheni]. In TARISZnYás, Márton: Gyergyó történeti néprajza, 31-44. Budapest: Akadémiai.

TóTH, Antónia

2009 Népi növényismeret az Örségben [Folk Plant Knowledge in the Örség Region]. Manuscript, Öriszentpéter.

TURNER, Nancy J.

1988 "The importance of a rose": evaluating the cultural significance of plants in Thompson and Lillooet Interior Salish." American Anthropologist 90:272290.

Dániel Babai is a research fellow at the Institute of Ethnology, Research Centre for the Humanities (ELKH). As an ethnoecologist, trained in both biology and cultural anthropology, he is interested in human-nature interactions, especially in extensive land-use management, sustainable forms of natural resource management, and related traditional ecological knowledge in the Carpathian Basin. E-mail: babai.daniel@btk.mta.hu

Mátyás Szépligeti is a ranger at the Örség National Park Directorate. His profession involves the monitoring, conservation, and management of protected natural values in the Őrség region. E-mail: matyas.szepligeti@onpi.hu

Antónia Tóth is an Örség-born environmental researcher. Her studies focus on the traditional land-use system in the Örség region, and especially on grassland management and the ethnobotanical knowledge of the local farmers. E-mail: tothantonia@gmail.com

Viktor Ulicsni is a research fellow at the Centre for Ecological Research, Institute of Ecology and Botany. He is an ethnozoologist and ecologist working in the Carpathian Basin and Moldova. E-mail: ulicsni.viktor@ecolres.hu

Open Access. This is an open-access article distributed under the terms of the Creative Commons Attribution 4.0 International License (https://creativecommons.org/licenses/by/4.0), which permits unrestricted use, distribution, and reproduction in any medium, provided the original author and source are credited, a link to the CC License is provided, and changes - if any - are indicated. (SID_1) 